\title{
A first estimate of the Milky Way dark matter halo spin
}

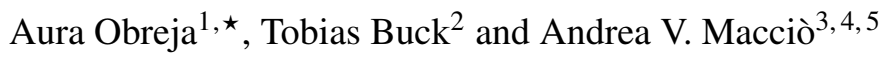 \\ 1 Universitäts-Sternwarte München, Scheinerstraße 1, D-81679 München, Germany \\ ${ }^{2}$ Leibniz-Institut für Astrophysik Potsdam (AIP), An der Sternwarte 16, D-14482 Potsdam, Germany \\ ${ }^{3}$ New York University Abu Dhabi, PO Box 129188, Saadiyat Island, Abu Dhabi, United Arab Emirates \\ ${ }^{4}$ Center for Astro, Particle and Planetary Physics $\left(\mathrm{CAP}^{3}\right)$, New York University Abu Dhabi \\ 5 Max-Planck-Institut für Astronomie, Königstuhl 17, D-69117 Heidelberg, Germany
}

Received April 1, 2021; accepted September 27, 2021

\begin{abstract}
The spin, or normalized angular momentum $\lambda$, of dark matter halos in cosmological simulations follows a log normal distribution and has little correlation with galaxy observables such as stellar masses or sizes. There is currently no way to infer the $\lambda$ parameter of individual halos hosting observed galaxies. Here, we present a first attempt to measure $\lambda$ starting from the dynamically distinct disks and stellar halos identified in high-resolution cosmological simulations with the Galactic Structure Finder (gsf). In a subsample of NIHAO galaxies analyzed with gsf, we find tight correlations between the total angular momentum of the dark matter halos, $J_{\mathrm{h}}$, and the azimuthal angular momentum, $J_{\mathrm{z}}$, of the dynamical distinct stellar components of the form: $\log \left(J_{\mathrm{h}}\right)=\alpha+\beta \cdot \log \left(J_{\mathrm{z}}\right)$. The stellar halos have the tightest relation with $\alpha=9.50 \pm 0.42$ and $\beta=0.46 \pm 0.04$. The other tight relation is with the disks, for which $\alpha=6.15 \pm 0.92$ and $\beta=0.68 \pm 0.07$. While the angular momentum is difficult to estimate for stellar halos, there are various studies that calculated $J_{\mathrm{z}}$ for disks. In application to the observations, we used Gaia DR2 and APOGEE data to generate a combined kinematics-abundance space, where the Galaxy's thin and thick stellar disks stars can be neatly separated and their rotational velocity profiles, $v_{\phi}(R)$, can be computed. For both disks, $v_{\phi}(R)$ decreases with radius with $\sim 2 \mathrm{~km} \mathrm{~s}^{-1} \mathrm{kpc}^{-1}$ for $R \gtrsim 5 \mathrm{kpc}$, resulting in velocities of $v_{\phi, \text { thin }}=221.2 \pm 0.8 \mathrm{~km} \mathrm{~s}^{-1}$ and $v_{\phi \text {,thick }}=188 \pm 3.4 \mathrm{~km} \mathrm{~s}^{-1}$ at the solar radius. We use our derived $v_{\phi \text {,thin }}(R)$ and $v_{\phi, \text { thick }}(R)$ together with the mass model for the Galaxy of Cautun et al. (2020) to compute the angular momentum for the two disks: $J_{\text {z,thin }}=(3.26 \pm 0.43) \times 10^{13}$ and $J_{z \text {,thick }}=(1.20 \pm 0.30) \times 10^{13} \mathrm{M}_{\odot} \mathrm{kpc} \cdot \mathrm{km} \cdot \mathrm{s}^{-1}$, where the dark halo is assumed to follow a contracted NFW profile. Adopting the correlation found in simulations, the total angular momentum of the Galaxy's dark halo is estimated to be $J_{\mathrm{h}}=2.69_{-0.32}^{+0.37} 10^{15} \mathrm{M}_{\odot} \mathrm{kpc} \cdot \mathrm{km} \cdot \mathrm{s}^{-1}$ and the spin estimate is $\lambda_{\mathrm{MW}}=0.061_{-0.016}^{+0.022}$, which translates into a probability of $21 \%$ using the universal $\log$ normal distribution function of $\lambda$. If the Galaxy's dark halo is assumed to follow a NFW profile instead, the spin becomes $\lambda_{\mathrm{MW}}=0.088_{-0.020}^{+0.024}$, making the Milky Way a more extreme outlier (with a probability of only $0.2 \%$ ).
\end{abstract}

Key words. Galaxy: fundamental parameters - structure - halo, galaxies: structure - kinematics and dynamics

\section{Introduction}

In the current mainstream model of structure formation, cold dark matter (DM) hierarchically clusters under the effect of gravity (Peebles 1965) into the so-called cosmic web (Bond et al. 1996) from the tiny density fluctuations present in the Universe at the epoch of recombination (Sachs \& Wolfe 1967, Silk 1968; Peebles 1982). In this scenario, gas cools and collapses into the potential wells provided by the density peaks of the cosmic web: the DM halos (White \& Rees 1978), where it starts forming stars. In the linear regime of perturbation growth, the evolution of structure formation can be described analytically. Once the perturbations grow into the non-linear phase, there are various approximations that can be used (Zel'Dovich 1970; Gunn \& Gott 1972; Gurbatov et al. 2012). A powerful alternative to these approximations is to trace structure formation either through $\mathrm{N}$ body simulations, which cover only DM and further apply semianalytic galaxy formation models (e.g., White \& Frenk 1991; Dalcanton et al. 1997; Mo et al. 1998), or through hydrodynamic

\footnotetext{
^obreja@usm.lmu.de
}

N-body simulations, which follow the coupled evolution of DM and baryons (e.g., Katz \& Gunn 1991; Katz et al. 1992; Katz 1992, Cen \& Ostriker 1992, Navarro \& White 1994, and the many works that have followed).

Early studies of large box N-body simulations have revealed that cold DM halos are self-similar (Navarro et al.|1997). Therefore, the density distributions of spherical DM halos can be completely described by only two parameters, namely: total mass, $M_{\mathrm{h}}$, and concentration, $c$. These two parameters are not independent. The correlation between them depends on the cosmological model assumed (e.g., Macciò et al. 2008) and this correlation also varies with redshift $z$ (e.g., Dutton \& Macciò 2014). The DM halo mass is an important predictor for fundamental galaxy properties such as total stellar mass and the star formation rate or star formation efficiency as probed by both analytic models (e.g., Peacock \& Smith 2000; van den Bosch et al. 2003, Vale \& Ostriker |2004; Conroy et al.|2007; Conroy \& Wechsler 2009; Guo et al. 2010; Moster et al. 2010, 2018; Behroozi et al. 2019) as well as cosmological hydrodynamical N-body simulations (e.g., Schaye et al. 2015; Wang et al. 2015). In observations, the masses and concentrations of DM halos can be 
constrained via models that Syer \& Tremaine (1996) generically referred to as "dynamical made-to-measure models" that include methods based on: distribution functions, moments (e.g., Jeans models), orbits (e.g., Schwarzschild orbit superposition method, Schwarzschild 1979), and particles (introduced by Syer \& Tremaine 1996, and now known as M2M).

Because cold DM can only interact gravitationally, the other important physical property of DM halos is the angular momentum, $J_{\mathrm{h}}$. In theory, a halo acquires its angular momentum through tidal torques induced by the misaligments between the inertia tensor of the collapsing Lagrangian patch of the halo and the large scale tidal field (tidal torque theory, TTT, Hoyle 1951; Doroshkevich 1970, Fall \& Efstathiou 1980, White 1984). In this scenario, most of the halo angular momentum (AM) is acquired at high $z$, where perturbations growth linearly (Catelan \& Theuns 1996). This linear growth phase of $J_{\mathrm{h}}$ ends when the Lagrangian patch of the spherical overdensity reaches its maximum expansion. At later times, mergers are the dominant processes that can alter $J_{\mathrm{h}}$ (e.g., Barnes \& Efstathiou 1987).

A fundamental assumption of TTT is that DM and baryons are tightly coupled, at least at high $z$, such that their specific AM are roughly equal: $j_{\text {baryon }} \simeq j_{\mathrm{h}}$. Another important assumption in galaxy formation models is that AM is conserved during the non-linear phase of perturbations growth. In this manner, the observed relation between galaxy sizes and their rotation velocities arises naturally by assuming the disk scale $R_{\mathrm{d}}$ to be equal to the virial radius $r_{\mathrm{h}}$ multiplied by the halo spin: $R_{\mathrm{d}} \sim \lambda r_{\mathrm{h}}$ (e.g., Dalcanton et al. 1997; Mo et al. 1998; Somerville et al. 2008; Firmani \& Avila-Reese 2009, Dutton et al. 2011).

Peebles (1971) introduced the spin $\lambda$, which is an adimensional measure of halo angular momentum: $\lambda=J|E|^{1 / 2} / G M^{5 / 2}$, where $J$ is the total (baryons $+\mathrm{DM}$ ) angular momentum of a halo, $E$ is the total energy, $M$ is the total mass, and $G$ is the gravitational constant. Using $\lambda$, halos of very different mass can be meaningfully compared. Early cold dark matter simulations resulted in median spin values $\bar{\lambda} \simeq 0.04-0.05$ (Barnes \& Efstathiou 1987, Efstathiou et al. 1988, Cole \& Lacey 1996). These simulations explored various cosmologies and found $\bar{\lambda}$ to be only mildly impacted by variations in the spectral index of the primordial power spectrum. Also, they found very weak trends for more massive halos to have lower $\lambda$.

Using more recent $\Lambda \mathrm{CDM}$ cosmological simulations, Bullock et al. (2001) showed that the probability distribution function of DM halo spins can be well fitted by a log-normal, with only slightly lower median $\lambda$ than the earlier results: $\bar{\lambda}=$ $0.042 \pm 0.006$. These authors also introduced a different definition of spin: $\lambda=J / M /\left(\sqrt{2} r_{\text {vir }} v_{\text {vir }}\right)$, where $r_{\text {vir }}$ and $v_{\text {vir }}$ are the virial radius and velocity, respectively, for which they found $\bar{\lambda}=0.035 \pm 0.005$ and a $\log$ width $\sigma_{\ln \lambda}=0.50 \pm 0.03$. This definition of spin has become the prefered one because it does not require an estimate of the total energy. Over the years, simulations have increased in size and resolution, but the $\lambda$ distribution remained stable, at least in terms of $\bar{\lambda}$; for instance, Jiang et al. (2019) obtained $\bar{\lambda}=0.037$ and $\sigma_{\ln \lambda}=0.215$. Furthermore, Benson (2017) showed that insufficient resolution leads to spurious high $\lambda$ values, which artificially increase $\sigma_{\ln \lambda}$. Therefore, the lower width of the distribution in more recent estimates can be attributed to significantly higher resolutions in these simulations $\left(\sim 10^{6}\right.$ particles per halo).

As expected from the anisotropic TTT (Codis et al. 2015), the DM halo spin directions correlate with the large scale environment of galaxies (e.g., Codis et al. 2012; López et al. 2021; Ganeshaiah Veena et al.2021). Nevertheless, the alignment sig- nal between the DM halo spin and the angular momentum of the galaxy disk is weak (e.g., Bailin et al. 2005, Bett et al. 2010, Debattista et al. 2015, Shao et al. 2016). Also, the modulus of halo spin $\lambda$ correlates very weakly or not at all with observable galaxy properties (or properties that can be derived from observations) such as (disk) size, stellar mass, or stellar angular momentum (e.g., Teklu et al. 2015, Rodriguez-Gomez et al.|2017, Desmond et al. 2017; Jiang et al. 2019; Behroozi et al. 2021). Hence, in semi-analytic models of galaxy formation (e.g., Somerville et al. 2008. Dutton et al.2011), the spin is assumed to be uncorrelated with any other galaxy property, its value for individual galaxies being simply drawn from the universal log-normal distribution. This lack of correlations with galaxy observables implies that $\lambda$, one of the fundamental properties of DM halos, cannot be inferred for individual observed galaxies.

In this study, we revisit the subsample of galaxies from the Numerical Investigation of a Hundred Astrophysical Objects (NIHAO, Wang et al. 2015), analyzed in Obreja et al. (2019), to look for correlations between the DM halo AM and the individual galactic stellar dynamical structures. Obreja et al. (2018. 2019) (hereafter Paper I and Paper II) showed that Gaussian mixture models applied to a stellar dynamical space of normalized $\mathrm{AM}$ and normalized binding energy are capable of disentangling a multitude of dynamical structures such as thin and thick disks, classical and pseudo bulges, and stellar halos. Therefore, a possible interesting direction of inquiry is to look for correlations between the build-up of the dark matter halos and the formation of robustly defined individual galaxy components. We find tight correlations between $J_{\mathrm{h}}$ and the stellar dynamical disks, and between $J_{\mathrm{h}}$ and the dynamical stellar halos. These relations allow us to infer the DM halo spin from galaxy properties that can be derived from observations, and we carried out such an exercise for the Milky Way (MW). We used a combination of MW observational data from Gaia (Gaia Collaboration et al. 2016), re-analyzed by Hogg et al. (2019), and Apache Point Observatory Galactic Evolution Experiment (APOGEE, Majewski et al. 2017), together with the Galaxy mass model of Cautun et al. (2020) and the circular velocity curve of Eilers et al. (2019) to compute the AM for the MW thin and thick stellar disk. Finally, by employing the relations found in simulations, we estimated the values of $J_{\mathrm{h}}$ and $\lambda$ for our Galaxy.

\section{Simulated galaxy sample}

NIHAO (Wang et al. 2015) is a collection of zoom-in hydrodynamical simulations run with the N-body smoothed particle hydrodynamics (SPH) code Gasoline2 (Wadsley et al. 2017) using a Planck cosmology (Planck Collaboration 2014). This simulation sample covers five orders of magnitude in stellar mass, from dwarfs to MW/M31 analogs with $\sim 10^{6}$ particles/halo in order to resolve the half mass radius of the galaxies. Star formation is implemented stochastically in dense $\left(\mathrm{n}>10.3 \mathrm{~cm}^{-3}\right)$ and cold $(\mathrm{T}>15000 \mathrm{~K})$ gas, with an efficiency of $10 \%$, such that a Kennicutt-Schmidt type relation is recovered. Two types of stellar feedback are included: SNe II blast-waves (Stinson et al. 2006) and photoheating of gas by massive stars before their SN stage (Stinson et al. 2013). The free parameters of the stellar feedback have been chosen such that one MW mass galaxy follows the abundance-matching stellar-to-halo mass relation (Moster et al. 2013; Behroozi et al. 2013; Kravtsov et al. 2018) throughout its evolution to $z=0$ (Stinson et al. 2013). With this fixed choice of feedback parameters, all NIHAO galaxies are within or very close to the permitted regions of abundance matching relations, both at low and at high $z \mathrm{~s}$ (Wang et al. 
2015). The simulations have been run assuming the initial mass function of Chabrier (2003), along with heavy elements enrichment from SNe Ia (Thielemann et al. 1986) and SNe II yields (Woosley \& Weaver 1995). Metals are allowed to diffuse as described in Wadsley et al. (2008). Gas is assumed to be in photoionization equilibrium with the $z$-dependent ultra violet background of Haardt \& Madau (2012), and to cool through metal lines and Compton scattering following Shen et al. (2010).

The DM halos are defined with the Amiga Halo Finder code (Gill et al. 2004, Knollmann \& Knebe 2009), such that the virial radius $r_{200}$ encloses a sphere with average density 200 times the cosmic critical matter density.

The NIHAO simulations have been tested extensively against observations, showing good agreement in terms of, for instance, the HI velocity function (Macciò et al. 2016, Dutton et al. 2019b), Tully-Fisher relation (Dutton et al. 2016b), properties of galactic satellites and field dwarf galaxies (Buck et al.2019a), stellar components properties (Obreja et al. 2016, 2018, 2019, Buck et al. 2020), properties of ultra diffuse dwarfs (Di Cintio et al. 2017).

Buck et al. (2020) analyzed MW-like galaxies in the simulated sample, run with both the fiducial NIHAO and increased resolutions. In the high-resolution sample, they find values for the disk scale heights between 0.2 and $0.4 \mathrm{kpc}$ for the thin disks, and 1.0 and $1.4 \mathrm{kpc}$ for the thick ones, as well as global scale lengths from Sérsic + exponential fits of the stellar surface mass density between 3.9 and $5.7 \mathrm{kpc}$. Stellar masses are only slightly lower in the higher-resolution runs, while scale heights are lower by an average of $25 \%$. In the Tully-Fisher relation (Figure 8 of Buck et al. 2020), NIHAO galaxies occupy the upper envelope, probably showing that they are a little too compact when compared with the observations. Nevertheless, when compared against MW (e.g., Bland-Hawthorn \& Gerhard 2016), they are in good agreement within their resolution limits. The rotation curves of the MW-like galaxies in the original NIHAO are shown in Fig. A.1 of Appendix A where the halo and stellar masses, as well as the spin parameters are also given. One simulated galaxy is particularly close to the MW's circular rotation curve $V_{\mathrm{c}}(R)$, as estimated by, for instance, Eilers et al. (2019), with the other MW-like galaxies wobbling around the observationally constrained $V_{\mathrm{c}}(R)$.

The stellar metallicities of NIHAO galaxies are in agreement with the observations of Gallazzi et al. (2005) and Panter et al. (2008) for stellar masses larger than $10^{9} \mathrm{M}_{\odot}$ (see Buck et al. 2021). Discrepancies are mostly restricted to the dwarf regime $\left(M_{*}<10^{9} \mathrm{M}_{\odot}\right)$, where both stellar and gas metallicities at fixed stellar mass tend to be lower in NIHAO than in the observations (McConnachie 2012, Berg et al. 2012; Kirby et al. 2013, Zahid et al. 2013). Considering the limited statistics of both simulations and observations, the large overlap between the above mentioned observations and the NIHAO galaxies indicate that these simulations have realistic metallicities at fixed stellar mas 1

The NIHAO simulations contributed to firmly establish that baryons can break the self similarity of DM halos, which develop cores in the halo mass ranges where star formation is most efficient (Tollet et al.|2016; Dutton et al. 2016a, 2017). This core formation effect predicted by Blumenthal et al. (1986) is seen in

\footnotetext{
1 Metallicity uncertainties for observed galaxies can be quite large, for instance, 0.7 dex differences depending on the calibration (Kewley \& Ellison 2008). Also, the vast majority of simulation works, including the ones cited here, do not measure metallicity in the same way as in observations because it would require costly simulation post-processing with radiation transfer codes. Thus, comparisons between simulations and observations should be taken with a grain of salt.
}

simulations that can resolve sufficiently high gas densities (e.g., Chan et al. 2015, Dutton et al. 2019a, 2020), with a strength depending on the stellar/halo mass ratio (e.g., Di Cintio et al. 2014, Tollet et al. 2016), and on the density threshold for star formation (e.g., Governato et al. 2012; Benítez-Llambay et al. 2019). Recently, the NIHAO sample has also been extended to massive elliptical galaxies after the implementation of super massive black hole formation, accretion, and feedback in the version of the Gasoline 2 code used to run the original sample (Blank et al. 2019); the addition of AGN feedback resolved the overcooling of the three most massive NIHAO halos.

In the current study, we used the subsample of 25 NIHAO galaxies from Wang et al. (2015), analyzed in Paper II. These galaxies have stellar masses between $7 \times 10^{8} \mathrm{M}_{\odot}$ and $2 \times 10^{11} \mathrm{M}_{\odot}$, and have been chosen to show no obvious signs of interactions in the stellar surface mass density maps at $z=0$. The following section gives a brief overview of Papers I and II, focusing on our definition of stellar galactic structures.

\section{Defining the dynamical stellar structures}

Historically, galaxies have been classified based on their disky or spheroidal photometric appearance, leading to the so-called Hubble diagram (Sandage 1961). With the recent advances in Integral Field Unit (IFU) spectrographs such as PPak at the Calar Alto Telescope (Kelz et al. 2006), Multi Unit Spectroscopic Explorer (MUSE) at the Very Large Telescope (VLT) (Bacon et al. 2010), Sydney-AAO Multi-object Integral-field spectrograph (SAMI) at the Anglo-Australian Telescope (Croom et al. 2012), or Mapping Nearby Galaxies at Apache Point Observatory (MaNGA) at Sloan (Bundy et al. 2015), the galaxy formation community has begun to broaden and refine the classification schemes, incorporating dynamical galaxy properties that can be extracted from the modeling of IFU data.

Unlike the observational data, simulations provide direct access to the full $6 \mathrm{D}$ position-velocity space of stellar particles through time. Therefore, in simulations, it is relatively easier to derive robust measures of intrinsic dynamical properties, such as the ratio of ordered to random motions (or rotational support $v / \sigma)$. One particularly straightforward and widely used method for looking at the extent to which a simulated galaxy is rotationally supported has been introduced by Abadi et al. (2003). These authors constructed the histograms of the stellar circularity parameter $\epsilon$ defined as the (specific) azimuthal $\mathrm{AM} j_{\mathrm{z}}$ normalized to the (specific) AM of a circular orbit with the same binding energy $j_{\mathrm{c}}: \epsilon=j_{\mathrm{z}} / j_{\mathrm{c}}$, where the $z$-axis is given by the direction of the stellar AM within the galaxy region $\left(\lesssim 0.2 r_{\text {vir }}\right)$. This parameter is $-1<\epsilon<1$, with counter-rotating particles having $\epsilon<0$, and disk particles clustering strongly toward $\epsilon \gtrless 1$. Therefore, assuming the spheroid or bulge to have no net rotation (corresponding to a symmetric $\epsilon$ distribution around 0 ), the dynamical bulge-to-total ratio can be computed as $B / T_{\text {dyn }}=2 \Sigma_{\mathrm{i}} M_{\mathrm{i}}\left(\epsilon_{\mathrm{i}}<0\right) / \Sigma_{\mathrm{i}} M_{\mathrm{i}}$, where $M_{\mathrm{i}}$ is the mass of particle, $i$. Thus, a good measure of rotational to velocity dispersion support becomes the corresponding dynamical disk-to-bulge ratio.

In order to get a less ambiguous assignment of particles as belonging to a disk or a spheroid or bulge, Doménech-Moral et al. (2012) employed a modified version of the kmeans clusterfinding algorithm in a 3D stellar kinematic space of circularity, normalized in(equatorial)-plane AM, $j_{\mathrm{p}} / j_{\mathrm{c}}$ (with total specific $\mathrm{AM}: \boldsymbol{j}=\boldsymbol{j}_{\boldsymbol{z}}+\boldsymbol{j}_{\boldsymbol{p}}$ ), and binding energy, $E$. In this way, they were able to separate not only the disk from the bulge, but also to pick up two different disk types: a thin and a thick one. 
In Paper I, we refined the method introduced by DoménechMoral et al. (2012), switching to Gaussian mixture models with unconstrained covariance instead of kmeans, in order to relax the latter algorithm's limitation to convex clusters of roughly equal weights. The 3D feature space that we use is $\left(j_{z} / j_{c}, j_{p} / j_{c}\right.$, $\left.e /|e|_{\max }\right)$, where the specific binding energy $e$ is normalized to the most bound stellar particle in the halo $|e|_{\max }$. This method is capable of disentangling a multitude of dynamical structures in simulated galaxies, including components that resemble observed thin or thick disks, classical or pseudo-bulges, and stellar halos (Paper II). The code we developed to select (in this manner) the dynamical components of simulated galaxies is called galactic structure finder (gsf) ${ }^{2}$. To get meaningful results, we tailored gsf to work on relaxed galaxies and to recompute the gravitational force at each stellar particle position in a galaxy by assuming its halo to be in isolation. In this context, "relaxed" means that the symmetry $z$-axis can be defined unambiguosly; the direction of the total stellar angular momentum computed within spheres of increasing radii has to vary little if at all with the radius. In this way, the normalized specific binding energy $e /|e|_{\max }$ is ensured to be well behaved for all stellar particles in a halo.

The relatively high resolution of the NIHAO simulations allowed us to go after dynamical galaxy components as faint as what is typically expected from stellar halos. To choose the optimal number of components, one straightforward strategy is to construct the functions Bayes Information Criteria (BIC, Schwarz 1978) or Akaike Information Criteria (AIC, Akaike 1974) versus the number of Gaussians. In the scikit-learn Python library (Pedregosa et al. 2011) that gsf uses for the clustering, the BIC is defined as: $B I C=-2 N \cdot \ln (L)+n_{\text {param }} \cdot \ln (N)$, and the $A I C$ is $A I C=-2 N \cdot \ln (L)+2 n_{\text {param }}$, where $N$ is the total number of samples (particles in this case), $\ln (L)$ is the per-sample average $\log$ likelihood, and $n_{\text {param }}$ is the number of free parameters, directly proportional to the number of Gaussian components $n_{\mathrm{k}}: n_{\text {param }}=10 n_{\mathrm{k}}-1$ for a $3 \mathrm{D}$ feature space and fully unconstrained covariance matrix. The large number of samples (stellar particles) $N$ to which we apply the Gaussian mixture models makes both of these criteria just scaled version of the log likelihood, since the penalty functions $n_{\text {param }} \cdot \ln (N)$ and $2 n_{\text {param }}$ are much smaller that the first $2 N \cdot \ln (L)$ terms. A typical curve $-\ln (L)$ vs $n_{\mathrm{k}}$ for a galaxy analyzed with gsf is shown in Buck et al. (2019b), and has no clear minimum that would permit selecting the "optimal" number of 3D Gaussians in a straightforward manner (however, also see Du et al. 2019, who used gsf with a heuristic BIC criteria to select the number of components in simulated galaxies).

The goal in Paper II was to identify stellar halos and separate disks if possible into thin and thick components. Therefore, visual inspection of models with similar $\ln (L) / B I C$ was necessary, given the large overlap in feature space of the various galaxy components and the small weight of stellar halos. The smaller (dwarf) galaxies in the sample have been separated into only two components: a disk and a spheroid, while the MW analogs in terms of stellar mass are made of up to five different dynamical structures (see their Figure A1). Table1] gives the positions of the following distinct dynamical components in the gsf input feature space: spheroid, classical bulge, pseudo bulge,

\footnotetext{
2 available at https://github.com/aobr/gsf. The new version of the code, gsf2, is available upon request from the main author. This version includes the possibility to choose other spaces for clustering, provides statistics for a wide range of physical parameters of each component, and uses an information criteria to automatically select the optimal number of components in a galaxy.
}

Table 1. Positions of the dynamical components in the feature space

\begin{tabular}{cccc}
\hline \hline Component & $j_{\mathrm{z}} / j_{\mathrm{c}}$ & $j_{\mathrm{p}} / j_{\mathrm{c}}$ & $e /|e|_{\max }$ \\
\hline spheroid & $0.05 \pm 0.13$ & $0.41 \pm 0.07$ & $-0.76 \pm 0.03$ \\
classical bulge & $0.02 \pm 0.08$ & $0.48 \pm 0.04$ & $-0.78 \pm 0.03$ \\
pseudo bulge & $0.18 \pm 0.09$ & $0.17 \pm 0.02$ & $-0.71 \pm 0.03$ \\
halo & $0.07 \pm 0.09$ & $0.35 \pm 0.06$ & $-0.39 \pm 0.04$ \\
disk & $0.68 \pm 0.08$ & $0.24 \pm 0.04$ & $-0.61 \pm 0.04$ \\
thin disk & $0.88 \pm 0.02$ & $0.18 \pm 0.03$ & $-0.61 \pm 0.03$ \\
thick disk & $0.64 \pm 0.05$ & $0.33 \pm 0.04$ & $-0.68 \pm 0.03$ \\
\hline
\end{tabular}

(stellar) halo, (single large-scale) disk, thin disk, and thick disk. Spheroids are very close to spherical symmetry, can have large radial extents, are very bound, have circularities peaking close to 0 , and show no traces of rotation in the line-of-sight edge-on perspective. Classical bulges are very close to spherical symmetry, very bound, very compact, have circularities peaking close to 0 , and show no traces of rotation in the line-of-sight edge-on perspective. Pseudo-bulges are flattened, compact, less bound than classical bulges and spheroids, and show signals of rotation in the line-of-sight edge-on perspective. Stellar halos are the least bound components, with very large radial extents, and usually show some signs of rotation (their peak circularities are slightly higher than 0). Single large-scale disks are flat, extended, with strong rotation patterns in the line-of-sight edge-on perspective, and their circularities peak at large values. Thin disks are very flat and extended, show strong rotation patterns in the line-ofsight edge-on perspective, and have the largest circularity peak - close to 1 - among the components of galaxies. Thick disk are less flat and extended than thin disks, show significant rotation patterns in the line-of-sight edge-on perspective, and their circularities peak at values lower than their corresponding thin disks. The corresponding values for all the components of all the galaxies in the sample are given in the table A1 of Paper II. The components' names have been chosen based on their surface mass density and line-of-sight velocity maps, as explained above, and on their positioning in the $\left(j_{z} / j_{c}, j_{p} / j_{c}, e /|e|_{\max }\right)$ space.

As already discussed in Paper II and shown in Table 1. the stellar dynamical components are well ordered in binding energies, from stellar halos, which are the least bound, passing through the disks, thin disks, thick disks, pseudo bulges, spheroids, and, finally, to classical bulges, which are the most bound structures. In terms of circularities, the most rotational supported components are the thin disks, followed by the disks, thick disks, and pseudo bulges, while the stellar halos, spheroids and classical bulges have almost no net rotation.

\section{4. $J_{h}$ vs the AM of stellar disks and halos}

In Paper II, we discussed at length the evolution of the specific AM for the various types of stellar structures present in the simulations. In particular, by tracing back in time the Lagrangian masses of the $z=0$ stellar structures and DM halos, we were able to assess the differences between the AM evolution of galactic components and that of their DM halos. Thus, it resulted that stellar disks (thin or thick, or single large-scale disks) have the most similar evolution to the DM halos (figure 12 in Paper II). On average, the material of $z=0$ disks reached its peak AM at redshifts $z_{\text {peak }} \sim 1.37$ and $\sim 1.65$ for thin and thick disks, respectively, and $z_{\text {peak }} \sim 2.11$ for single large-scale disks, while the material of the host halos peaked in $\mathrm{AM}$ at $z_{\text {peak }} \sim 1.44$. From $z_{\text {peak }}$ to $z=0$, disks and DM halos lose some part of the maximum AM reached, with the DM 


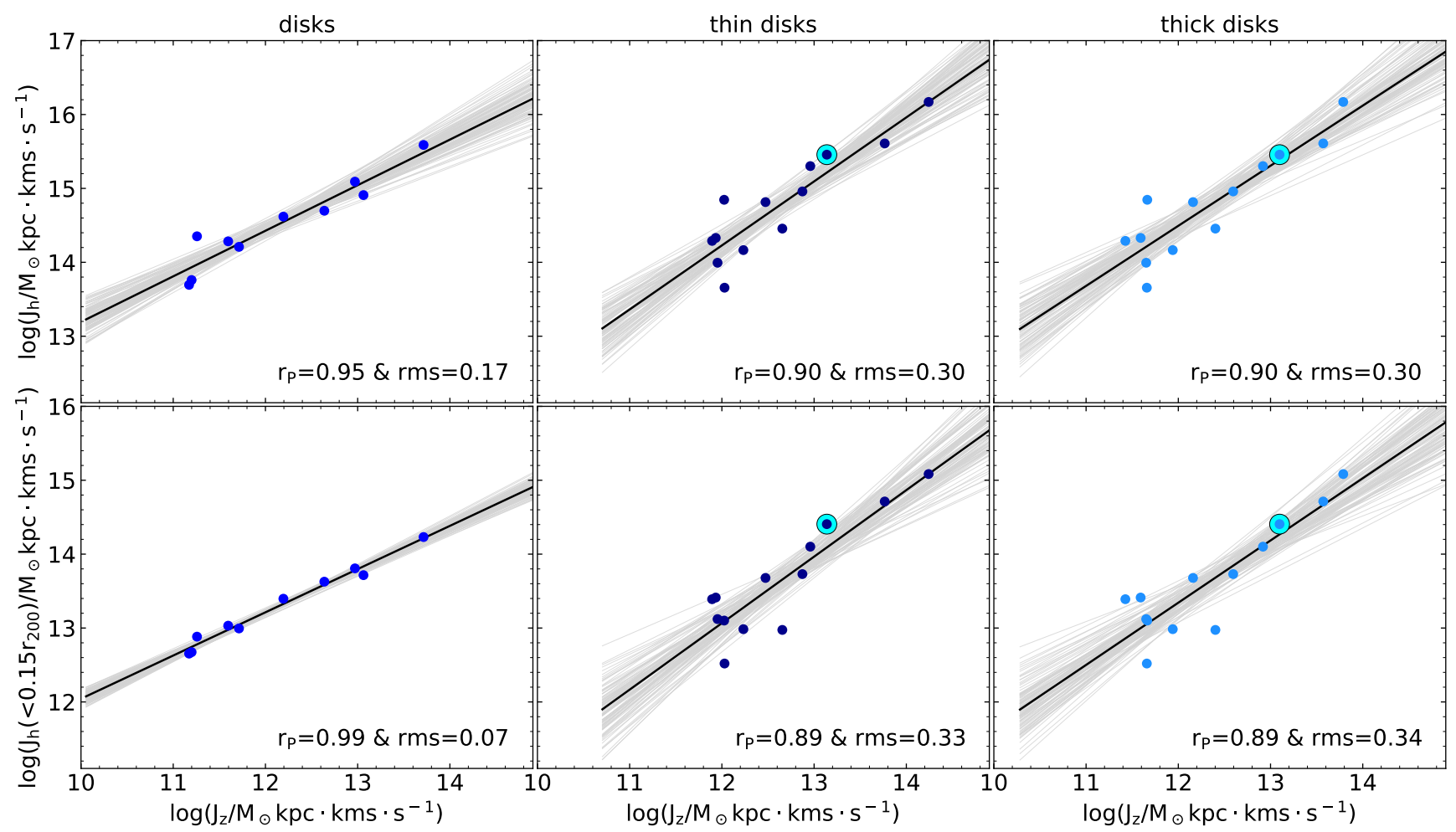

Fig. 1. Relations between the azimuthal AM of the dynamical disk components and the AM modulus of the DM halo for the galaxy sample in Paper II. In the top panels the y-axis gives the total DM AM within the virial radius $J_{\mathrm{h}}$, while the bottom ones show the AM within $15 \%$ of the virial radius $J_{\mathrm{h}}\left(<0.15 r_{200}\right)$. From left to right, the panels show the relations for (single large scale) disks, thin and thick, respectively. In each panel, the black line is the linear fit through the data points, while the gray lines show 100 realization of the relation drawn from the covariance matrix of the fit. The Pearson correlation coefficient $r_{\mathrm{P}}$ and the root-mean-square deviation $r m s$ are given in the bottom right corner of each panel. In the top and central panels, the highlighted cyan points correspond to the best MW analog in the NIHAO sample, g8.26e11 (Obreja et al.|2018, Buck et al. 2020).

halos retaining the most $f_{\mathrm{j}}=0.75 \pm 0.15$, and the thick disks the least $f_{\mathrm{j}}=0.41 \pm 0.15$, where $f_{\mathrm{j}}$ quantifies the retained fraction of angular momentum as the ratio between the (specific) $\mathrm{AM}$ at $z=0$ and the maximum specific (AM) reached during the universe's lifetime: $f_{\mathrm{j}}=j(z=0) / \max (j)$. By comparison, the dispersion-dominated structures (classical and pseudo bulges, and spheroids) have larger $z_{\text {peak }}$ and they lose most of their acquired AM, having an overall AM evolution that is not particularly similar to the DM one. Also, it is important to take into account that supernova feedback can remove low AM gas from galaxies (e.g., Dutton \& van den Bosch 2009; Brook et al. 2011), some of which will come back onto the symmetry plane at larger radii and with larger AM acquired in the likely spinning circumgalactic medium (e.g., Fraternali \& Binney 2006; Brook et al. 2012; Athanassoula et al. 2016; Peschken et al. 2017). This recycling of disk material prior to the star formation which happens preferentially in or very close to the symmetry plane, together with secular processes such as radial migration (Sellwood \& Binney 2002), means that it is not expected for stellar disks and dark matter to precisely share the same specific AM.

The similarities in AM evolution between the progenitor material of stellar disks and that of DM halos do indeed translate into relatively tight correlations at $z=0$ between the azimuthal AM component of stellar disks and the AM modulus of the DM halo, as shown in Fig. 11. The top panels give the total DM halo AM measured within the virial radius $r_{200}$, while the bottom ones the corresponding DM halo AM measured in the galaxy region $r<0.15 r_{200}$. We fitted all these individual relations with power laws:

$\log \left(J_{\mathrm{h}}\right)=\alpha+\beta \cdot \log \left(J_{\mathrm{Z}}\right)$,

which are depicted by the solid black lines in the panels of Fig. 1 where both $J_{\mathrm{h}}$ and $J_{\mathrm{z}}$ are measured in $\mathrm{M}_{\odot} \mathrm{kpc} \cdot \mathrm{km} \cdot \mathrm{s}^{-1}$. The Pearson correlation coefficient $r_{\mathrm{P}}$ as well as the root-mean-square deviation $r m s$ are quoted in each panel, and all the fit parameters are given in Table 2. We chose to plot on the $\mathrm{x}$-axis the azimuthal component of the stellar AM, given that this is the quantity that can be estimated from observations (for disks $J \cong J_{\mathrm{z}}$ ), while $J_{\mathrm{h}}$ plotted on the y-axis is the quantity we want to estimate.

When looking at the total DM halo AM within the virial radius $J_{\mathrm{h}}$, the tightest correlation appears for the single disks $\left(r_{\mathrm{P}}=0.95\right.$ and $\left.r m s=0.17\right)$. We attribute this behavior to the nearly perfect coevolution of angular momenta of single disks' progenitor material and DM halos' progenitor material up to $z \bumpeq 2$, as shown by Fig. 12 in Paper II (blue versus thick black). The thin and thick disks have a slightly different high- $z$ AM history than the DM halos, translating into a less tight correlation and larger scatter, even if their low- $z$ evolution is more similar to that of DM halos than to that of single disks. If the AM of the DM halos is measured within the galaxy region $\left(r<0.15 r_{200}\right)$, the correlation with the single disks is very close to ideal $\left(r_{\mathrm{P}}=0.99\right.$ bottom-left panel of Fig. 1), while for the thin and thick disk, it is slightly worse (central and right bottom panels versus central 


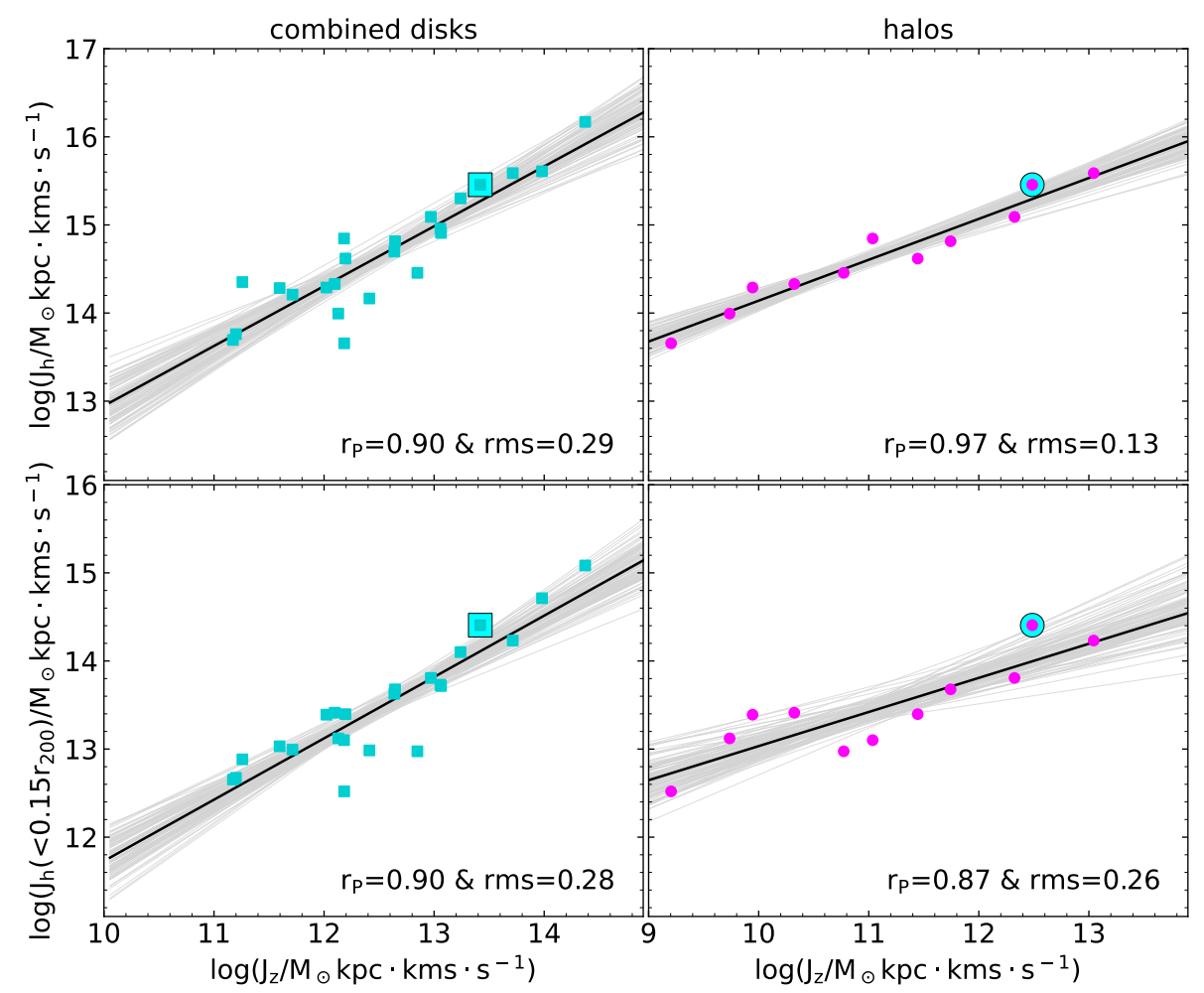

Fig. 2. Relations between the azimuthal AM of the dynamical disk components and the AM modulus of the DM halo for the galaxy sample in Paper II. Same details as in Fig. 1. but for all the dynamical disks combined (left) and the stellar halos (right). In the left panels, each galaxy with a double disk is represented by a single point with abscissa $J_{z}=J_{z, \text { thin }}+J_{z, \text { thick}}$. The highlighted cyan points in all panels correspond to the best MW analog in the NIHAO sample, g8.26e11.

Table 2. Best-fit parameters for the relations between the AM of the DM halo and that of the stellar dynamical components.

\begin{tabular}{c|cccc|cccc}
\hline \hline & \multicolumn{4}{|c|}{$\log \left(J_{\mathrm{h}}\right)=\alpha+\beta \cdot \log \left(J_{\mathrm{z}}\right)$} & \multicolumn{3}{c|}{$\log \left(J_{\mathrm{h}}\left(<0.15 r_{200}\right)\right)=\alpha+\beta \cdot \log \left(J_{\mathrm{Z}}\right)$} \\
\hline Component & $\alpha$ & $\beta$ & $r_{\mathrm{P}}$ & $r m s$ & $\alpha$ & $\beta$ & $r_{\mathrm{P}}$ & $r m s$ \\
\hline halos & $9.50 \pm 0.42$ & $0.46 \pm 0.04$ & 0.97 & 0.13 & $9.16 \pm 0.82$ & $0.39 \pm 0.07$ & 0.87 & 0.26 \\
disks & $7.03 \pm 0.88$ & $0.62 \pm 0.07$ & 0.95 & 0.17 & $6.19 \pm 0.35$ & $0.58 \pm 0.03$ & 0.99 & 0.07 \\
thin disks & $3.83 \pm 1.58$ & $0.87 \pm 0.13$ & 0.90 & 0.30 & $2.27 \pm 1.75$ & $0.90 \pm 0.14$ & 0.89 & 0.33 \\
thick disks & $4.74 \pm 1.47$ & $0.81 \pm 0.12$ & 0.90 & 0.30 & $3.24 \pm 1.64$ & $0.84 \pm 0.13$ & 0.89 & 0.34 \\
combined disks & $6.15 \pm 0.92$ & $0.68 \pm 0.07$ & 0.90 & 0.29 & $4.77 \pm 0.91$ & $0.70 \pm 0.07$ & 0.90 & 0.28 \\
\hline
\end{tabular}

and right top ones). These differences in behavior between single and double stellar disk galaxies is worth looking into more detail in a future study, since they possibly encode information on why some objects develop one instead of two disk components.

In observations, it is not always straightforward or possible to classify a galaxy as having either a thin + thick disk or a single disk (e.g., Elmegreen et al. 2017). This fact set together with our small galaxy sample size naturally leads to the left panels of Fig. 2. where we considered the single and double (thin + thick) disks together in order to increase the statistics. The parameters of the power law fits shown in this figure are also given in Table 2 The relation between the AM of stellar disks and that of their DM halos has a direct application in observations; many works have already quantified the AM of large samples of stellar disks in order to look at how the AM scales with the stellar mass (e.g., Fall \& Efstathiou 1980, Fall \& Romanowsky |2018; Romanowsky \& Fall 2012: Obreschkow \& Glazebrook 2014, Posti et al. 2018). In this manner, spin distribution for observed galaxy samples could be computed and compared with the predictions from simulations. For instance, Teklu et al. (2015) found that halos hosting disk-dominated galaxies have the log-normal distribution of DM halo spins skewed toward larger $\lambda$ than the full DM halo population $(0.064$ vs 0.043$)$. In the next section, we apply the relation for combined disks to MW to estimate the spin of its host halo, and leave for a future work deriving the $\lambda$ distribution for large samples of observed galaxies.

In the right panel of Fig. 2, we show the only tight correlation we found within the group of dispersion dominated stellar components, namely between the stellar halos azimuthal AM and their DM halos. Actually, at the virial scale, the DM halo AM can be most accurately predicted from the $J_{\mathrm{Z}}$ of stellar halos $\left(r_{\mathrm{P}}=0.97\right.$ and $\left.r m s=0.13\right)$. The main reason for this correlation is the comparable spatial extent of stellar and DM halos. As can be appreciated from Fig. 2, and from the differences in Table 2 (between the normalization of the relation involving the complete DM halo extent and the galaxy region only), most of the contribution to $J_{\mathrm{h}}$ comes from radii $R>0.15 r_{200}$. Therefore, it is natural that the AM of the extended stellar halos would closely 
predict the AM of the extended DM ones. Though it is the tightest, the relation between the stellar and DM halos is harder to use in observations, because it requires: i) accurate velocity tracers all throughout a very faint galaxy component that can possibly extend all the way to the virial radius, and ii) accurate mass distributions for the dynamically distinct stellar halo.

\section{Application to MW}

The MW is now known to host a large diversity of substructure thanks to the many observational campaigns that targeted its stars, such as: Radial Velocity Experiment (RAVE, Steinmetz et al. 2006), APOGEE (Allende Prieto et al. 2008), Sloan Extension for Galactic Understanding and Exploration (SEGUE, Yanny et al. 2009), Large Sky Area Multi-Object Fibre Spectroscopic Telescope (LAMOST, Deng et al.2012), Gaia-ESO Survey (Gilmore et al. 2012), GIRAFFE Inner Bulge Survey (GIBS Zoccali et al.2014), or Galactic Archaeology with the HERMES survey (GALAH, De Silva et al. 2015). The main body of stars of the Galaxy are structured into: a nuclear star cluster (Becklin \& Neugebauer 1968), a boxy/peanut bulge (Okuda et al. 1977), a bar (Hammersley et al.2000), a thin and a thick disk (Gilmore \& Reid 1983), and a stellar halo (Searle \& Zinn 1978). There are some indications that the Galaxy might also host a small classical bulge (e.g., discussion in the review by Bland-Hawthorn \& Gerhard 2016). While the MW's proximity allows the mapping of individual stars, and an unmatched level of detail with respect to other galaxies, it also obfuscates estimates of some key parameters such as the scale lengths of the thin and thick disk.

The thick disk of the MW separates from the thin one in various properties. The thick disk has a larger scale height (e.g., Jurić et al. 2008), older stellar ages (e.g., Gilmore et al. 1995) and it is less rotationally supported (e.g., Soubiran et al. 2003), more metal poor (Fuhrmann 1998), and more $\alpha$ element-enhanced (e.g., Fuhrmann 1998; Bensby et al. 2003; Reddy et al. 2006) than the thin disk.

For the purpose of deriving the AM and spin of MW's DM halo, we need to first compute the AM of its thin and thick stellar disks. Under the assumption of axis symmetry, $J_{\mathrm{z}}$ can be computed in observations as:

$J_{\mathrm{z}}=\int_{0}^{\infty} \int_{-\infty}^{\infty} \rho(R, z) v_{\phi}(R, z) 2 \pi R^{2} d R d z$

where $R$ and $z$ are cylindrical coordinates, $\rho(R, z)$ is the 3D mass density distribution, and $v_{\phi}(R, z)$ is the rotational velocity. For galaxy disks, a usual assumption is that $\rho(R, z)$ takes the form:

$\rho(R, z)=\frac{\Sigma_{0}}{2 z_{d}} \exp \left(-\frac{R}{R_{d}}\right) \exp \left(-\frac{|z|}{z_{d}}\right)$,

with $R_{d}$ and $z_{d}$ as the radial and vertical scale lengths and $\Sigma_{0}$ the normalization of the face-on exponential surface mass density profile. Under the additional assumption that the rotational velocity does not depend on the height above the plane $v_{\phi}(R, z)=v_{\phi}(R)$, Eq. 2 becomes:

$J_{\mathrm{z}}=\frac{M}{R_{d}^{2}} \int_{0}^{\infty} v_{\phi}(R) e^{-R / R_{d}} R^{2} d R$,

where $M=2 \pi R_{d}^{2} \Sigma_{0}$ is the total disk mass.

Therefore, to compute the AM of the MW's disk in the approximation of Eq. 4, we need the total mass $M$, exponential scale length, $R_{d}$, and the rotational velocity profile,$v_{\phi}(R)$. For both the thin and thick disk of the MW, we can take the total mass and scale length from previous published works. Therefore, in order to compute $J_{z \text {,thin }}$ and $J_{z \text {,thick }}$, we only need to construct the velocity profiles $v_{\phi \text {,thin }}(R)$ and $v_{\phi \text {,thick }}(R)$.

\subsection{Thin and thick disks of the MW}

To separate the MW's stellar disk into a thin and a thick component, we used the sample of red-giant branch stars with parallaxes re-estimated by Hogg et al. (2019). These authors used stars from a cross-matched catalog of APOGEE, Gaia, Two Micron All-Sky Survey (2MASS, Skrutskie et al. 2006) and Widefield Infrared Survey Explorer (WISE, Wright et al. 2010) to construct a data-driven model capable of predicting parallaxes with less than $15 \%$ uncertainties, therefore improving on the values available from Gaia. Their model implicitly accounts for reddening and extinction, and results in precise distances to stars with less than $10 \%$ uncertainties up to $20 \mathrm{kpc}$ from the Sun. These characteristics make this sample ideal to construct dynamical models of the Galaxy (e.g., Eilers et al. 2019, 2020, Cautun et al. 2020), and are very well suited for our purpose of computing the rotational velocity profiles for the thin and thick disks.

One way to separate MW's thin disk from the thick one would be to apply a clustering algorithm in the same parameter space as the one used by gsf. This approach, however, is complicated by the fact the Galaxy's mass models still have large uncertainties, and these models are needed to compute the stellar binding energies. If we restrict ourselves only to the circularity parameter, $j_{\mathrm{z}} / j_{\mathrm{c}}$, we can use circular velocity curves $V_{\mathrm{c}}(R)$ from literature to compute the normalization, $j_{\mathrm{c}}$. For this purpose, we used the circular velocity curve published by Eilers et al. (2019), who derived it by fitting a Jeans model to the star sample with improved parallaxes of Hogg et al. (2019), and radial velocities and positions from APOGEE. Therefore, we approximate $j_{\mathrm{c}} \cong V_{\mathrm{c}} R$, where $V_{\mathrm{c}}$ is the circular velocity at the radial position, $R$, of the star in the galactic plane. The circular velocity curve of Eilers et al. does not extend to $R<5 \mathrm{kpc}$ because their modeling cannot properly account for the presence of the bar (e.g., Wegg et al. 2015). For this reason, we separate the thin from the thick disk only in the radial range $R>5 \mathrm{kpc}$. Given the extensive evidence that the MW's disk stars show a bimodality in their $\alpha$-enhancement (e.g., Fuhrmann 1998; Bensby et al. 2003), we chose $[\mathrm{O} / \mathrm{Fe}]$ from the APOGEE catalog as second feature for the clustering. We chose oxygen as a tracer of $\alpha$-enhancement for two reasons: i) it results in a clean separation of the two MW disk components and ii) it facilitates comparisons with our previous simulations, where only oxygen and iron are traced in detail. Using other elements (e.g., magnesium) or combinations of $\alpha$ elements available in APOGEE does not change the conclusions of this study.

To convert the positions and velocities of stars to galactocentric coordinates with $\mathrm{Sgr} \mathrm{A}^{*}$ as origin, we assumed: a position of the Sun $\left(x_{\odot}, y_{\odot}, z_{\odot}\right)=\left(R_{\odot}, 0, z_{\odot}\right) \mathrm{kpc}$, where $R_{\odot}=8.122 \mathrm{kpc}$ (Gravity Collaboration et al. 2018) and $z_{\odot}=0.025 \mathrm{kpc}(\mathrm{Ju}-$ rić et al. 2008), and a velocity of the $\operatorname{Sun}\left(v x_{\odot}, v y_{\odot}, v z_{\odot}\right)=$ $(-11.1,245.8,7.8) \mathrm{km} \cdot \mathrm{s}^{-1}$ (Reid \& Brunthaler 2004), which is the same as in Eilers et al. (2019).

We made further selections to the cross-matched APOGEE and Hogg et al. (2019) catalogs to best suit our purpose. Thus, we chose only: i) prograde stars with $0<v_{\phi}<350 \mathrm{~km} \mathrm{~s}^{-1}$, ii) confined within $1 \mathrm{kpc}$ from the galactic plane $|z|<1 \mathrm{kpc}$, and iii) with positive defined errors for $[\mathrm{O} / \mathrm{Fe}]$. The first two selection criteria ensure we are minimizing the contamination from the stellar halo. The circular velocity curve of Eilers et al. (2019) 


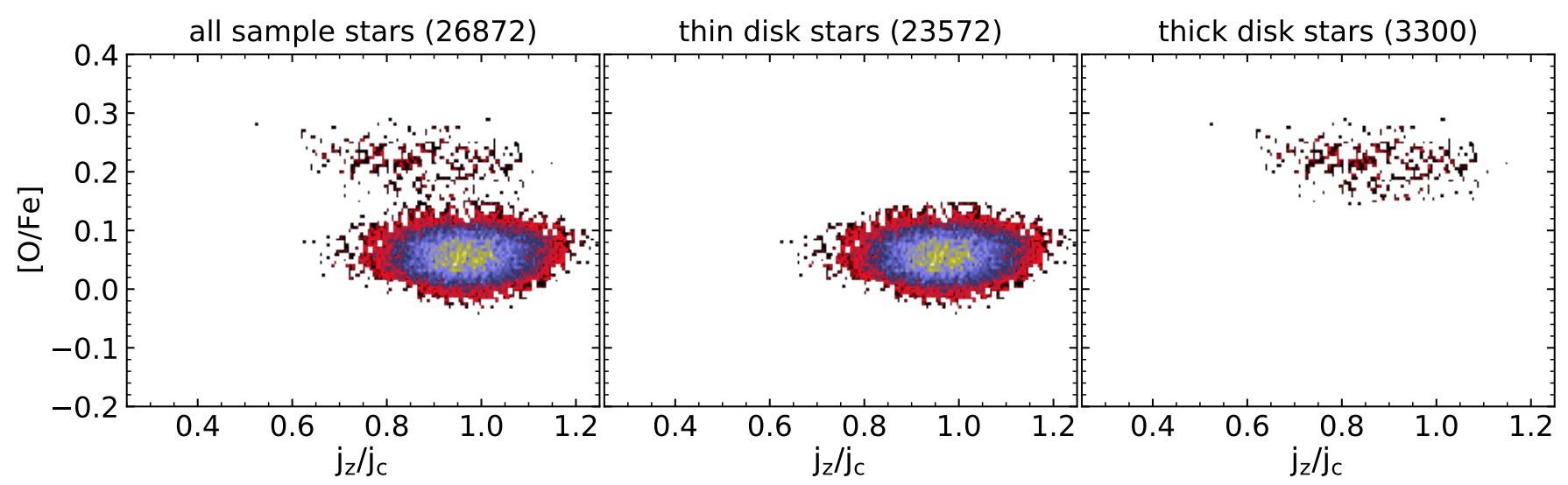

Fig. 3. $\mathrm{MW}$ disk separation in the feature space $\left(j_{\mathrm{z}} / j_{\mathrm{c}},[\mathrm{O} / \mathrm{Fe}]\right)$ using Gaussian Mixture clustering with two components. The observational data are a subsample of the stars in Hogg et al. (2019) for which the circularity parameter $j_{\mathrm{z}} / j_{\mathrm{c}}$ could be estimated. We approximate $j_{\mathrm{c}} \cong V_{\mathrm{c}} R$, where $V_{\mathrm{c}}$ is the circular velocity at the radial position $R$ of the star in the galactic plane, and $V_{\mathrm{c}}(R)$ is taken from (Eilers et al. 2019).

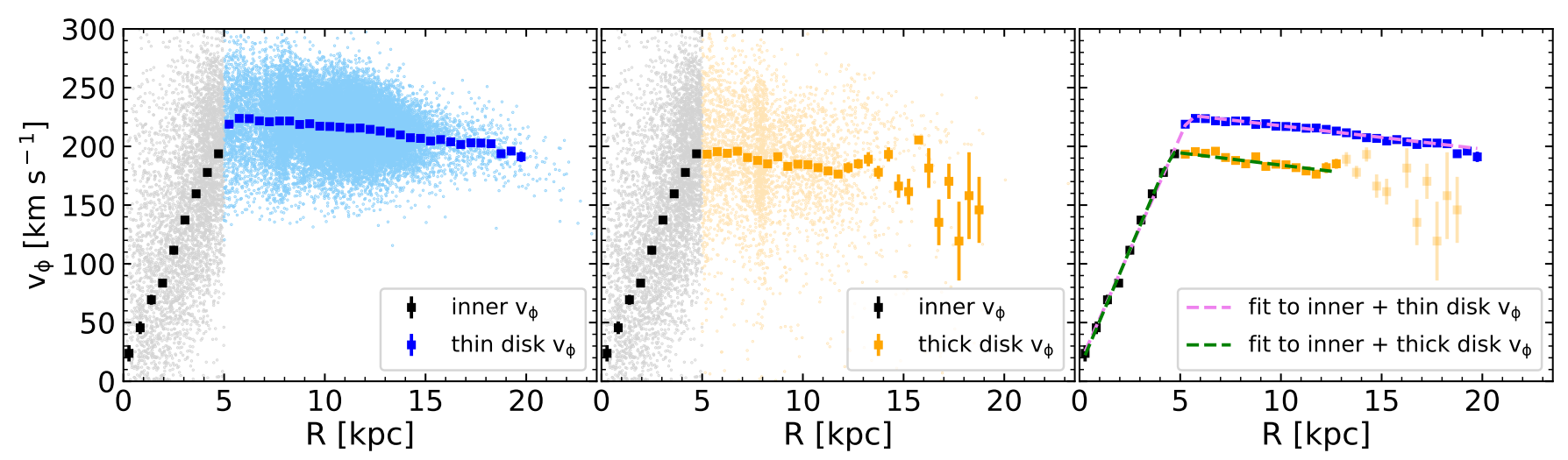

Fig. 4. Rotational velocity profiles for the MW's disks. The gray points in the left and central panels are the stars with $|z|<1 \mathrm{kpc}$ and $\left|v_{\phi}\right|<$ $350 \mathrm{~km} \mathrm{~s}^{-1}$, which were used to construct the inner $v_{\phi}$ profile. The light blue and orange points in the left and central panels, respectively, are the thin- and thick-disk stars selected in the $\left(j_{z} / j_{\mathrm{c}},[\mathrm{O} / \mathrm{Fe}]\right)$ space. The parameters of the two fits in the right panel are given in Table 3 For the thick disk, only the region $R<13 \mathrm{kpc}$ was considered for the fit.

does not extend to $R<5 \mathrm{kpc}$, further reducing the sample for clustering to 27044 stars. Figure 3 shows the separation of the selected sample of stars in the feature space $\left(j_{\mathrm{z}} / j_{\mathrm{c}},[\mathrm{O} / \mathrm{Fe}]\right)$ in form of $2 \mathrm{D}$ histograms. To define the two clusters visible in the complete sample on the left panel we used the Gaussian Mixture clustering of scikit-learn with two components. This clustering method is able to separate highly imbalanced clusters. The central and right panels show the $2 \mathrm{D}$ histograms of the two clusters identified as the thin and thick disks, respectively. Given that the stars are clearly separated in this space, other methods such as hierarchical clustering lead to a very similar assignment of stars to one group or the other.

Once the stars are assigned to either the thin or the thick disk, we can construct, for both components, the rotational velocity profiles $v_{\phi}(R)$ as ensemble averaged values in fixed radial bins of $\Delta R=0.5 \mathrm{kpc}$ for $5 \leqslant R \leqslant 20 \mathrm{kpc}$. As done in Eilers et al. (2019), we estimate the uncertainties on $v_{\phi}(R)$ by bootstrapping with 100 samples. The rotational velocity of disk stars is expected to get to 0 at $R=0$. Therefore, to extend the two curves below $5 \mathrm{kpc}$, we construct the $v_{\phi}(R)$ for all stars in the cross-matched catalog with $|z|<1 \mathrm{kpc}$ and $\left|v_{\phi}\right|<350 \mathrm{~km} \mathrm{~s}^{-1}$, and take it to represent both the thin and the thick disk rotation. This is a reasonable assumption considering the fact that exponential disks (as assumed in the mass model of Cautun et al.2020) should have $v_{\phi} \rightarrow 0$ as $R \rightarrow 0$. Figure 4 shows the derived $v_{\phi}(R)$ profiles for the thin (solid blue squares, left panel) and thick (solid orange squares, central panel) disks. Both profiles are declining with radius according to $\sim 2 \mathrm{~km} \mathrm{~s}^{-1} \mathrm{kpc}^{-1}$. In the solar neighborhood $R=R_{\odot}$, the resulting $v_{\phi}$ values for the thin and thick disks of 221.2 \pm 0.8 and $188 \pm 3.4 \mathrm{~km} \mathrm{~s}^{-1}$ are close to the values derived by Haywood et al. (2013): $\sim 220$ and $170 \pm 16 \mathrm{~km} \mathrm{~s}^{-1}$. This means that at $R_{\odot}$, the thick disk is lagging behind the thin one by $\sim 33 \mathrm{~km} \mathrm{~s}^{-1}$.

The solid black squares in all panels of Fig. 4 give the $v_{\phi}(R)$ for $R<5 \mathrm{kpc}$, computed as explained above. We fit the two observational profiles (solid black + solid blue representing the $v_{\phi}(R)$ of the thin disk, and solid black + solid orange representing the $v_{\phi}(R)$ of the thick disk) with double linear fits:

$v_{\phi}(R)= \begin{cases}v_{0}+\gamma_{0} \cdot R, & \text { if } R<R_{\mathrm{break}} \\ v_{1}+\gamma_{1} \cdot R, & \text { if } R \geqslant R_{\mathrm{break}},\end{cases}$

where continuity at the break radius $R_{\text {break }}$ implies that $v_{0}=$ $v_{1}+\left(\gamma_{1}-\gamma_{0}\right) \cdot R_{\text {break }}$. The right panel of Fig. 4 shows together the fits (dashed violet or green curves) and data (black and blue or orange squares) for both disks (thin or thick), and Table 3 gives the values of the parameters in Eq. 5. The sample thick disk stars cover very sparsely regions at large radii and, therefore, the fit for the thick disk was limited to the data with $R<13 \mathrm{kpc}$ (solid vs faint orange in the right panel of Fig. 4). We did not force the fit to pass through the origin, and 


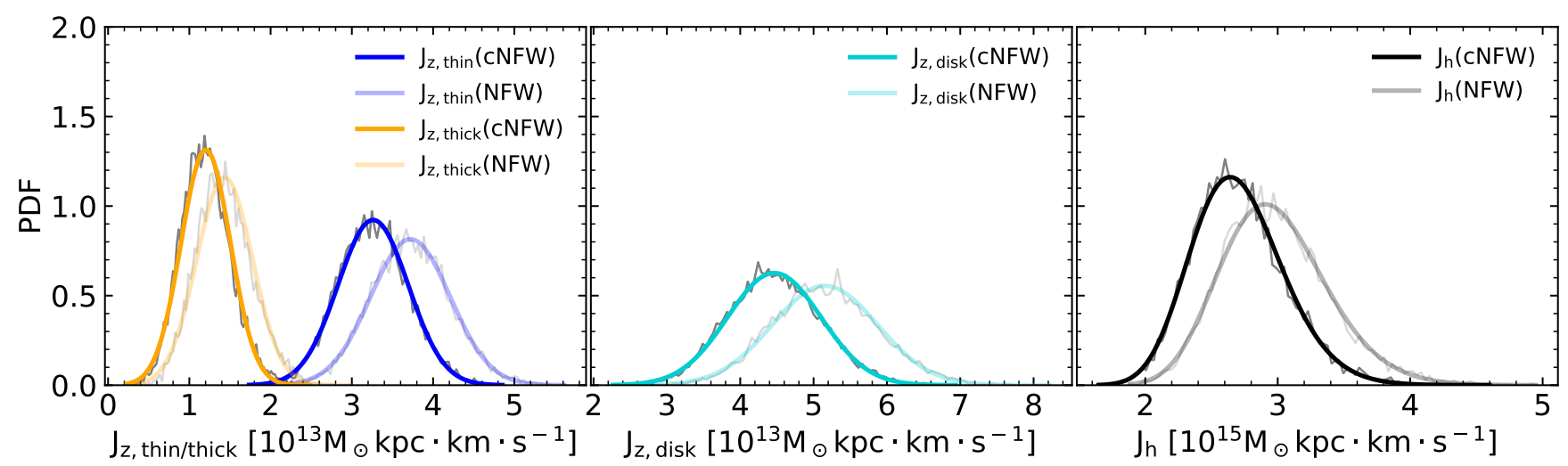

Fig. 5. MW angular momentum PDFs for its: thin and thick disks (left), combined stellar disk (center), and DM halo (right). The underlying PDFs in thin black are well approximated by normal distributions for the stellar components (thick blue, orange, and turquoise curves) and by log normal for the DM ones (thick black and gray curves). Solid or faint colors show the predictions for the contracted or uncontracted NFW mass model, respectively.

Table 3. Best-fit parameters for the MW's rotational velocity profiles.

\begin{tabular}{ccc}
\hline \hline Component & thin disk & thick disk \\
\hline$R_{\text {break }}[\mathrm{kpc}]$ & $5.47 \pm 0.03$ & $4.56 \pm 0.06$ \\
$\gamma_{0}\left[\mathrm{~km} \mathrm{~s}^{-1} \mathrm{kpc}^{-1}\right]$ & $38.73 \pm 0.66$ & $40.43 \pm 1.05$ \\
$v_{1}\left[\mathrm{~km} \mathrm{~s}^{-1}\right]$ & $237.30 \pm 0.65$ & $204.86 \pm 2.41$ \\
$\gamma_{1}\left[\mathrm{~km} \mathrm{~s}^{-1} \mathrm{kpc}^{-1}\right]$ & $-1.98 \pm 0.06$ & $-2.08 \pm 0.29$ \\
\hline
\end{tabular}

as a consequence $v_{0}>0$ for both disks. Setting $R_{\text {break }}=5 \mathrm{kpc}$ and $v_{0}=0$ for both disks and fitting only the second branch in Eq. 5. we get $v_{1, \text { thin }}=236.71 \pm 0.64 \mathrm{~km} \mathrm{~s}^{-1}, \gamma_{1, \text { thin }}=$ $-1.93 \pm 0.06 \mathrm{~km} \mathrm{~s}^{-1} \mathrm{kpc}^{-1}, v_{1, \text { thick }}=206.93 \pm 3.14 \mathrm{~km} \mathrm{~s}^{-1}$, and $\gamma_{1, \text { thick }}=-2.30 \pm 0.35 \mathrm{~km} \mathrm{~s}^{-1} \mathrm{kpc}^{-1}$, where the continuity condition at $R_{\text {break }}$ gives $\gamma_{0}=\gamma_{1}+v_{1} / R_{\text {break }}$. In this case there is a more marked difference between the velocity decline with radius, $\gamma_{1, \text { thin }}$ vs $\gamma_{1, \text { thick }}$. Table B.1 in Appendix B gives our derived $v_{\phi \text {,hin }}(R)$ and $v_{\phi, \text { thick }}(R)$, shown in Fig. 4

\subsection{AM of the MW stellar disks and DM halo}

For the MW's mass distribution by components, we applied the recent model from Cautun et al. (2020). These authors consider the following baryonic components: a bulge, a thin and a thick stellar disk, a gas disk (HI + molecular), and a gaseous halo, each with its own functional form. In their model, all the bulge parameters except total mass, the exponential scale heights of the two stellar disks, and all the gas disk parameters are fixed to previously derived values (Jurić et al. 2008, McMillan 2017). The mass profile of their gaseous halo is derived from simulations (Schaye et al.|2015; Sawala et al.|2016; Grand et al.|2017, 2019), such that it has no free parameters, and the total mass in this component is just a function of the total virial mass (dark matter + baryons). Therefore, the free parameters for the baryonic components in their model remain: total thin disk mass $M_{\text {thin }}$ and scale length, $R_{\text {thin }}$, total thick disk mass, $M_{\text {thick }}$ and scale length, $R_{\text {thick }}$, and total bulge mass, $M_{\text {bulge }}{ }^{3}$. For the DM distribution, Cautun et al. (2020) considered two possibilities: an uncontracted and a contracted Navarro-Frenk-White (NFW) profile (Navarro et al. 1997). Their Bayesian model is constrained by the MW's ro-

\footnotetext{
3 The actual free parameters used by Cautun et al. (2020) are the surface mass density normalizations $\Sigma_{0 \text {,thin }}$ and $\Sigma_{0, \text { thick }}$, and the volume mass density normalization $\rho_{0}$ for the bulge.
}

tation curve of Eilers et al. (2019), the virial mass estimate of Callingham et al. (2019), the ratio between thin and thick disks densities at $R_{\odot}$ from the Gravity Collaboration et al. (2018), and the vertical force at $(R, z)=\left(R_{\odot}, 1.1 \mathrm{kpc}\right)$ of Kuijken \& Gilmore (1991), and slightly favors the contracted NFW profile. They discuss the predictions of their two models with different DM profiles to other observables such as total stellar disk mass, escape velocity at $R_{\odot}$, and stellar-to-DM mass ratio, concluding that these extra constrains also favor the contracted profile, but not definitively.

To compute the angular momentum for the thin and thick disks, we use both of the models from Cautun et al. (2020) along with the best-fit parameters from their Table 2. We symmetrize their reported uncertainties, such that we can use a multivariate normal distribution with a covariance that approximates the most relevant correlations in their Fig. 12 to propagate the uncertainties of their mass models into our estimates for the disks' AM. Table C. 1 th summarizes the parameters we adopted from Cautun et al. (2020) and the assumed covariances are given in Appendix C In practice, we generated 10000 samples from the three independent covariance matrices of the AM, mass and the velocity models involved in Eqs. 1. 4, and 5. To compute the AM of the DM halo, we used Eq. 1 with the best-fit parameters for combined disks in Table 2. Figure 5 shows the resulting probability distributions functions (PDFs) of AM for the thin and thick disks (left), combined stellar disk (center), and DM halo (right) as thin black curves in each panel. The colored curves in the figure are the normal (left and central panel) and log-normal (right panel) distributions generated from the first and second order moment of the corresponding PDFs. The larger masses for the thin and thick disks in the uncontracted NFW model naturally lead to larger estimates for the stellar AM of the disks, $3.72 \pm 0.49$ vs $3.26 \pm 0.43 \times 10^{13} \mathrm{M}_{\odot} \mathrm{kpc} \cdot \mathrm{km} \cdot \mathrm{s}^{-1}$ for the thin disk and $1.43 \pm 0.34$ vs $1.20 \pm 0.30 \times 10^{13} \mathrm{M}_{\odot} \mathrm{kpc} \cdot \mathrm{km} \cdot \mathrm{s}^{-1}$ for the thick disk, and propagate into a larger AM of the DM halo, namely: $2.96_{-0.37}^{+0.43}$ versus $2.69_{-0.32}^{+0.37} \times 10^{15} \mathrm{M}_{\odot} \mathrm{kpc} \cdot \mathrm{km} \cdot \mathrm{s}^{-1}$. Table 4 gives the $\mathrm{AM}$ and specific $\mathrm{AM}$ for the stellar disk, its two components, and the DM halo.

\footnotetext{
4 The uncertainty in $J_{\mathrm{h}}$ (as well as in $j_{\mathrm{h}}$ and $\lambda_{\mathrm{MW}}$ implicitly) takes into account only the covariance and not the scatter of the $J_{\mathrm{h}}=f\left(J_{\text {disk }}\right)$ relation. Considering the 0.29 dex scatter of this relation, the values for $J_{\mathrm{h}}, j_{\mathrm{h}}$ and $\lambda_{\mathrm{MW}}$ should be considered certain within a factor of $\sim 2$.
} 
Table 4. AM of MW's stellar disks and DM halo. The highlighted rows indicate properties whose values depend on the relation between the $\mathrm{AM}$ of disks and DM halos from simulations. The rest of the values are derived directly from the observational data alone.

\begin{tabular}{ccc}
\hline \hline Property & contracted NFW & NFW \\
\hline$J_{\text {z,thin }}\left[10^{13} \mathrm{M}_{\odot} \mathrm{kpc} \cdot \mathrm{km} \cdot \mathrm{s}^{-1}\right]$ & $3.26 \pm 0.43$ & $3.72 \pm 0.49$ \\
$J_{\mathrm{z} \text {,thick }}\left[10^{13} \mathrm{M}_{\odot} \mathrm{kpc} \cdot \mathrm{km} \cdot \mathrm{s}^{-1}\right]$ & $1.20 \pm 0.30$ & $1.43 \pm 0.34$ \\
$J_{\mathrm{z} \text {,disk }}\left[10^{13} \mathrm{M}_{\odot} \mathrm{kpc} \cdot \mathrm{km} \cdot \mathrm{s}^{-1}\right]$ & $4.46 \pm 0.64$ & $5.15 \pm 0.72$ \\
$J_{\mathrm{h}}\left[10^{15} \mathrm{M}_{\odot} \mathrm{kpc} \cdot \mathrm{km} \cdot \mathrm{s}^{-1}\right]$ & $2.69_{-0.32}^{+0.37}$ & $2.96_{-0.37}^{+0.43}$ \\
$j_{\mathrm{z} \text {,hin }}\left[\mathrm{kpc} \cdot \mathrm{km} \cdot \mathrm{s}^{-1}\right]$ & $1026 \pm 59$ & $935 \pm 51$ \\
$j_{\text {z,thick }}\left[\mathrm{kpc} \cdot \mathrm{km} \cdot \mathrm{s}^{-1}\right]$ & $1308 \pm 242$ & $1343 \pm 216$ \\
$j_{z, \text { disk }}\left[\mathrm{kpc} \cdot \mathrm{km} \cdot \mathrm{s}^{-1}\right]$ & $1089^{-1} \pm 2$ & $1021 \pm 62$ \\
$j_{\mathrm{h}}\left[\mathrm{kpc} \cdot \mathrm{km} \cdot \mathrm{s}^{-1}\right]$ & $2769_{-485}^{+589}$ & $3617_{-546}^{+643}$ \\
$\lambda_{\mathrm{MW}}$ & $0.061_{-0.016}^{+0.022}$ & $0.088_{-0.020}^{+0.024}$ \\
\hline
\end{tabular}

To place the MW in the broader context of nearby galaxies, we can look to, for instance, the Spitzer Photometry \& Accurate Rotation Curves sample (SPARC, Lelli et al. 2016), which is a compilation of 175 galaxies with good quality rotation curves derived from previously published $\mathrm{HI}$ and $\mathrm{H}_{\alpha}$ data and with good photometry in the Spitzer $3.6 \mu \mathrm{m}$ band. In particular, Posti et al. (2018) used 92 galaxies from SPARC (with large enough radial coverage of the velocity profile and inclinations $>30^{\circ}$ ) to argue that the specific AM of (stellar) disks is an unbroken powerlaw of disk mass over more than four orders of magnitudes in mass, from dwarfs to massive spirals. Interestingly, Posti et al. (2018) find a relatively small scatter for this relation, of only 0.15 dex. Placing the Galaxy in the $j_{\text {disk }}-M_{\text {disk }}$ plane, where $M_{\text {disk }}=M_{\text {thin }}+M_{\text {thick }}$, we find it to be $1.7 \sigma$ below the relation of Posti et al. for the uncontracted NFW model, and $1.1 \sigma$ below for the contracted one. Therefore, if we take the relation of Posti et al. at face value, the contracted NFW model is favored over the uncontracted one.

To place the MW's DM halo in context, we computed the spin following the definition of Bullock et al. (2001):

$\lambda=\frac{J_{h} / M_{h}}{\sqrt{2} r_{200} v_{200}}$,

where the virial velocity is $v_{200}=\sqrt{G M_{200} / r_{200}}, M_{200}$ is the total virial mass, and $G$ is the gravitational constant. To compute the virial radius $r_{200}$ as the radius enclosing an average overdensity 200 times the critical matter density, we assume the following cosmological parameters from Planck Collaboration et al. (2020): $h=0.6737, \Omega_{\mathrm{m}}=0.3147$ and $\Omega_{\Lambda}=0.6853$, and the total halo mass $M_{200}$ of the MW corresponding to the contracted and uncontracted NFW profiles.

The values of the MW's halo spin for the two DM mass models are reported in Table 4, and are shown in Fig. 6 together with the universal $\log$ normal distribution as quantified by Jiang et al. (2019): $\bar{\lambda}=0.037$ and $\sigma_{\ln \lambda}=0.215$. The large uncertainty in $\lambda_{\mathrm{MW}}$ is mostly driven by the uncertainties in the mass models of Cautun et al. (2020): reducing all the uncertainties in the mass models by a factor of 10 result in an uncertainty that is about four times lower 5

The contracted NFW model results in a spin of $\lambda_{\mathrm{MW}}=$ $0.061_{-0.016}^{+0.022}$, which is $2.3 \sigma_{\ln \lambda}$ away from the peak of the log normal distribution. In other words, the probability for a halo to have

\footnotetext{
5 Forcing the fit to the velocity profiles to $R=5 \mathrm{kpc}$ and $v_{0}=0 \mathrm{~km} \mathrm{~s}^{-1}$ changes the values in table 4 by $\sim 1 \%$ or less.
}

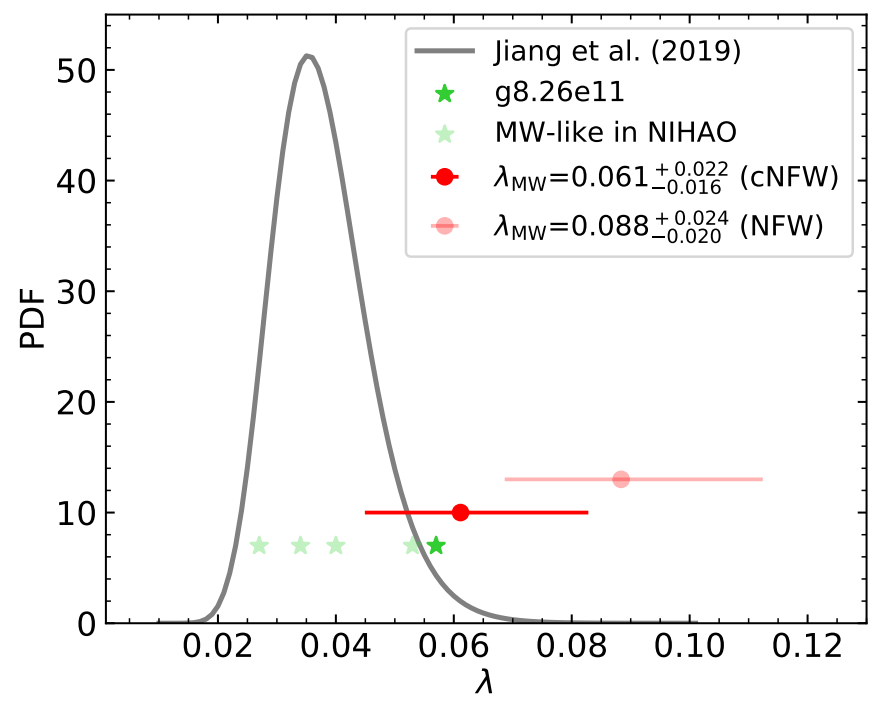

Fig. 6. DM halo spin distribution for the NIHAO sample (gray curve, Jiang et al. 2019), together with the MW predictions for the NFW and contracted NFW mass models (faint and solid red points). The green stars mark the spins of the MW-type galaxies in NIHAO, among which galaxy g8.26e11 is the closest analog, both in terms of stellar and DM mass, as well as in terms of stellar disk structure (Obreja et al. 2018. Buck et al. 2020). Most of the uncertainty in $\lambda_{\mathrm{MW}}$ comes from the uncertainties in the mass models of Cautun et al. (2020). The ordinates of the red and green points have no physical meaning.

this value of $\lambda$ (within its uncertainty) is $21 \%$. For the uncontracted NFW model, the resulting spin $0.088_{-0.020}^{+0.024}$ is a more extreme outlier, $4.0 \sigma_{\ln \lambda}$ away from the peak, which translates into a probability of only $0.2 \%$. This means that under our assumption that the AM of the DM halos can be derived from the AM of their stellar disks, the contracted NFW model for the MW's DM halo is strongly favored over the uncontracted one. In any case, both estimates of $\lambda_{\mathrm{MW}}$ imply that MW's DM halo spins significantly faster than what is expected for halos of similar masses.

To get an idea of how reasonable our derived value of $\lambda_{\mathrm{MW}}=$ 0.061 is, we also show in Fig. 6 the spins of MW-type galaxies in NIHAO (green stars). From left to right, the five green stars represent the simulated galaxies: $\mathrm{g} 7.66 \mathrm{e} 11(\lambda=0.027), \mathrm{g} 6.96 \mathrm{e} 11$ $(\lambda=0.034), \mathrm{g} 7.08 \mathrm{e} 11(\lambda=0.040) \mathrm{g} 7.55 \mathrm{e} 11(\lambda=0.053)$ and g8.26e11 $(\lambda=0.057)$. Figure A.1 in Appendix A shows the circular velocity curves of these simulated galaxies in comparison with MW data. The last simulated galaxy, g8.26e11, which has a spin parameter very close to the one inferred for the Galaxy has already been shown to be a very good MW analog in terms of DM halo mass, total stellar mass, and structure of the thin and thick stellar disks (Paper I, Buck et al. 2020). This simulated galaxy had its last important merger $\sim 10 \mathrm{Gyr}$ ago. The DM mass ratio of this event is 5.7:1, and this merger is responsible for $\sim 60 \%$ of the $z=0$ DM halo's AM (Fig. 8 in Paper I). Both the timing and the mass ratio of this merger are close to the values inferred for the event that is thought to have triggered Gaia Sausage (major merger 8 to 10 Gyr ago, Belokurov et al. 2018) also known as Gaia Enceladus (4:1 merger ratio 10 Gyr ago, Helmi et al. 2018).

The uncanny resemblance of g8.26e11 to MW raises the possibility that $\lambda$ should also be one of the important parameters (together with the $z=0$ halo mass and its mass accretion history) when selecting halos from DM-only simulations to resimulate at higher resolution; the goal here is that they would 
end up hosting MW analogs that are as good a fit as possible. For instance, if we consider the stellar mass function $\Phi\left(M_{*}\right)$ as computed from the combination of Galaxy And Mass Assembly survey (GAMA, Wright et al. 2017) and Bernardi et al. (2013), with a lower limit of $10^{8} \mathrm{M}_{\odot}$, we get a $1 \%$ probability ${ }^{6}$ of picking up a galaxy with the MW's stellar mass $\left(5.04_{-0.52}^{+0.43} \times 10^{10} \mathrm{M}_{\odot}\right.$, as in the cNFW model of Cautun et al.). Given that $\lambda$ does not depend on $M_{*}$, in a survey (or in a large scale hydrodynamical simulation) complete down to stellar masses of $10^{8} \mathrm{M}_{\odot}$, the probability of finding a galaxy with the MW's stellar mass and the MW's spin would be $0.22 \% 7$. The results of this study are based on a limited number of galaxies, but in combination with other highresolution simulations of MW analogs from the literature (e.g., Sawala et al. 2016, Grand et al. 2017, 2019, Garrison-Kimmel et al. 2018, Libeskind et al. 2020; Applebaum et al. 2021) the sample size could be increased. At the same time, comparing results across different physics implementations can gauge the detailed impact of baryonic physics.

The MW is known as a rather atypical spiral galaxy also because of its two largest satellites: the Large and Small Magellanic Clouds (LMC and SMC). Configurations of the type MWLMC-SMC are thought to be quite rare (e.g., Boylan-Kolchin et al.2011; González et al. 2013; Wang et al.2021), but the probability of a LMC-mass satellite is higher in MW-M31 galaxy pairs (e.g., Santos-Santos et al. 2021). The exact orbital history of these two satellites is still under debate, but currently it has been well established that LMC and SMC are on prograde orbits, close to the MW's plane (e.g. D'Onghia \& Fox 2016). Irrespective of the detailed infall scenarios for LMC and SMC, it is very likely that these mergers brought in a significant amount of AM, making the MW's DM halo a highly spinning structure. Finally, having an estimate of the DM halo's AM for the MW opens up the possibility of including the dark halo rotation in dynamical models of our Galaxy.

\section{Conclusions}

Using a subsample of simulated galaxies from the NIHAO project, we show that the total dark matter halo angular momentum, $J_{\mathrm{h}}$, is tightly correlated with the azimuthal angular momentum, $J_{\mathrm{z}}$, of dynamical disks and stellar halos. We use the data driven definition of dynamical stellar structures from Obreja et al. (2018, 2019), where Gaussian mixture models are applied in the parameter space of normalized angular momentum - normalized binding energy.

The tightest correlation is with the dynamical stellar halos, which is not unsurprising given that dark matter and stellar halos share similar spatial extensions. The other important correlation we find is between $J_{\mathrm{h}}$ and the $J_{\mathrm{z}}$ of dynamical stellar disks, albeit with a larger scatter and a smaller correlation coefficient than for stellar halos $\left(r m s=0.29\right.$ dex and $r_{\mathrm{P}}=0.90$ vs $r m s=0.13 \mathrm{dex}$ and $\left.r_{\mathrm{P}}=0.97\right)$. This relation can be explained by the fact that the angular momentum evolution of the Lagrangian masses forming the $z=0$ stellar disks and their host dark matter halos are relatively similar. The larger scatter in this relation can be at least partially explained by angular momentum redistribution suffered by the progenitor gas of $z=0$ stellar disks as an effect of stellar feedback. All parameters of the power laws between $J_{\mathrm{h}}$ and $J_{\mathrm{z}}$ are given in Table 2

We think the reasons why we do find tight relations between the dark matter halo and stellar components angular momenta,

\footnotetext{
${ }^{6} p\left(M_{\mathrm{MW}}\right)=\int_{M_{\mathrm{MW}, \text { low }}}^{M_{\mathrm{MW}, \text { pp }}} \Phi\left(M_{*}\right) d M_{*} / \int_{10^{8} \mathrm{M}_{\odot}}^{\infty} \Phi\left(M_{*}\right) d M_{*}$

${ }^{7} p\left(M_{*}, \lambda\right)=p\left(M_{*}\right) p(\lambda)$
}

while many other previous studies did not, are twofold: i) our data-driven definition of stellar dynamical structures is robust and realistic, and ii) we look at the physical property that is known to be conserved (at least to some degree), namely, the angular momentum - and not at the specific angular momentum or spin parameter. Disks are expected to conserve a large part of their initial or maximum angular momentum, similar to the case of dark matter halos, and in contrast to bulges. Therefore, if we have a good definition of what a "disk" is, as in which stellar particles and stars belong to the "dynamical" disk in a simulated or a real galaxy, we can more accurately test how closely disks follow the angular evolution of dark matter halos. And if such disks do follow the dark matter, we expect to find tighter correlations between the angular momenta of the two than in cases without a robust and physically meaningful definition for a disk. Our second reason is entirely related to the scatter in the relations between: i) halo mass and stellar mass (inverted $M_{\mathrm{star}}-M_{\mathrm{h}}$ relations, e.g., Moster et al. 2018); ii) disk scale length and stellar mass (e.g., Lelli et al. 2016); and iii) disk-to-total ratio and total stellar mass (e.g., Lang et al. 2014). The scatter in these three relations hinders any correlation between the spin or the specific angular momentum of dark matter halos and galaxy properties that can be derived from observations, such as stellar mass, disk mass, disk scale length, disk specific angular momentum, or stellar and baryonic spin.

In particular, the relation between $J_{\mathrm{h}}$ and $J_{\mathrm{z} \text {,disk }}$ can be applied in a straightforward manner with current observational data to derive angular momenta of dark matter halos hosting real galaxies, and, subsequently, to compute their spin parameters $\lambda$. We carried out such an exercise for the Milky Way using observational data from APOGEE and Gaia DR2, with improved parallaxes from Hogg et al. (2019), the circular velocity curve estimate of Eilers et al. (2019), and the Galaxy mass model of Cautun et al. (2020).

In the parameter space of circularities, namely, $\alpha$ enhancement, $j_{\mathrm{z}} / j_{\mathrm{c}}-[\mathrm{O} / \mathrm{Fe}]$, the sample of stars from Hogg et al. (2019) occupies two distinct but partially overlapping regions, which can be separated using clustering analysis (e.g., Gaussian mixtures) and subsequently associated with MW's thin and thick disks. This separation allows us to construct the rotational velocity profiles for the two populations of stars, $v_{\phi \text {,thin }}(R)$ and $v_{\phi \text {,thin }}(R)$. For radii $R \gtrsim 5 \mathrm{kpc}$, we find both disks to be well described by linear relations $v_{\phi}(R)=v_{1}+\gamma_{1} \cdot R$ with similar slopes $\gamma_{1} \simeq-2 \mathrm{~km} \mathrm{~s}^{-1} \mathrm{kpc}^{-1}$, but different normalizations, that is, $v_{1 \text {,thin }}=237.3 \pm 0.7 \mathrm{~km} \mathrm{~s}^{-1}$ and $v_{1 \text {,thick }}=204.9 \pm 2.4 \mathrm{~km} \mathrm{~s}^{-1}$. These relations imply an almost constant velocity lag of the thick disk with respect to the thin one of $\sim 33 \mathrm{~km} \mathrm{~s}^{-1}$ (measured at $R_{\odot}$ ) for $R \gtrsim 5 \mathrm{kpc}$, and result in rotational velocities at the Sun's radius of $221.2 \pm 0.8 \mathrm{~km} \mathrm{~s}^{-1}$ and $188 \pm 3.4 \mathrm{~km} \mathrm{~s}^{-1}$ for the thin and thick disks, respectively.

We calculated the angular momenta for the thin and the thick disks, $J_{z \text {,thin }}$ and $J_{z \text {,thick }}$ by convolving the derived $v_{\phi \text {, thin }}(R)$ and $v_{\phi \text {,thin }}(R)$ with the corresponding exponential mass profiles from Cautun et al. (2020). We used both MW mass models from Cautun et al. (2020), one assuming a contracted NFW profile for the dark matter halo and the other an uncontracted NFW. Thus, for the contracted and uncontracted NFW profiles, the two disks' angular momenta in units of $10^{13} \mathrm{M}_{\odot} \mathrm{kpc} \cdot \mathrm{km} \cdot \mathrm{s}^{-1}$ are: $J_{z \text {,thin }}=3.26 \pm 0.43 / 3.72 \pm 0.49$ and $J_{\text {z,thick }}=1.20 \pm 0.30 / 1.43 \pm 0.34$, and together they translate into an angular momentum for the dark matter halo of $J_{\mathrm{h}}=$ $2.69_{-0.32}^{+0.37} / 2.96_{-0.37}^{+0.43} \times 10^{15} \mathrm{M}_{\odot} \mathrm{kpc} \cdot \mathrm{km} \cdot \mathrm{s}^{-1}$. Factoring in the virial masses of the models for the contracted and uncontracted dark matter profiles, the spin parameter $\lambda_{\mathrm{MW}}$ is estimated to be 
$0.061_{-0.016}^{+0.022} / 0.088_{-0.020}^{+0.024}$, which is $2.3 \sigma_{\ln \lambda} / 4.0 \sigma_{\ln \lambda}$ away from the peak of the universal log normal spin distribution, as quantified by Jiang et al. (2019). In other words, the probability of a halo having a spin within the range derived for the NFW model is a factor of ten smaller than the probability corresponding to the $\lambda$ range of the contracted NFW $(0.2 \%$ vs $21 \%)$. Therefore, from the estimate of $\lambda_{\mathrm{MW}}$, the contracted NFW model for the Galaxy is strongly favored over the uncontracted one. Comparing MW's spin estimate with spins of MW-type galaxies in NI$\mathrm{HAO}$, we find that our best Galaxy analog (very similar halo and stellar masses, Fig. A.1 in the Appendix A also has a very similar parameter $\lambda=0.057$ to $\lambda_{\mathrm{MW}}$. Interestingly, this particular simulated galaxy experienced a major merger with similar characteristics (5.7:1 dark matter halo ratio $\sim 10 \mathrm{Gyr}$ ago) as the one thought to be responsible for the Gaia Enceladus feature (4:1 merger ratio 10 Gyr ago, Helmi et al. 2018). Also, this merger event in the simulation is responsible for a significant fraction of the final angular momentum of this galaxy's dark matter halo.

Relations such as the ones we found between the dynamical stellar components of galaxies and their host dark matter halos open up the possibility to compute the spin distribution for observed galaxies and compare it to the now well-established global distribution from simulations. In a more general sense, having estimates for the dark matter angular momenta of observed galaxies provides a unique new constraint on their formation histories and a closer glimpse into the properties of dark matter halos in the real universe.

Acknowledgements. We thank the anonymous referee for their comments which helped improve this manuscript. We would also like to thank Fabrizio Arrigoni Battaia for the many discussions on this work, and to the Lorentz Center in Leiden for hosting the workshop Dynamical reconstruction of galaxies, where many ideas pivotal to this work have been discussed. All figures have been made with matplotlib (Hunter 2007). We have also used the Python libraries numpy (Walt et al.2011), scipy (Eric Jones et al. 2001), scikit-learn (Pedregosa et al.2011), pandas (Wes McKinney 2010), and astropy (Astropy Collaboration et al. 2013 2018). F2PY (Peterson 2009) and pynbody (Pontzen et al. 2013) have been used in parts of the simulation analysis. This research was carried out on the High Performance Computing resources at New York University Abu Dhabi; on the ISAAC cluster of the Max-Planck-Institut für Astronomie and on the HYDRA clusters at the Rechenzentrum in Garching. We greatly appreciate the contributions of these computing allocations. AO is funded by the Deutsche Forschungsgemeinschaft (DFG, German Research Foundation) - 443044596. TB acknowledges support from the European Research Council under ERC-CoG grant CRAGSMAN-646955.

\section{References}

Abadi, M. G., Navarro, J. F., Steinmetz, M., \& Eke, V. R. 2003, ApJ, 597, 21 Akaike, H. 1974, IEEE Transactions on Automatic Control, 19, 716

Allende Prieto, C., Majewski, S. R., Schiavon, R., et al. 2008, Astronomische Nachrichten, 329, 1018

Applebaum, E., Brooks, A. M., Christensen, C. R., et al. 2021, ApJ, 906, 96

Astropy Collaboration, Price-Whelan, A. M., Sipőcz, B. M., et al. 2018, AJ, 156, 123

Astropy Collaboration, Robitaille, T. P., Tollerud, E. J., et al. 2013, A\&A, 558, A33

Athanassoula, E., Rodionov, S. A., Peschken, N., \& Lambert, J. C. 2016, ApJ, 821,90

Bacon, R., Accardo, M., Adjali, L., et al. 2010, in Society of Photo-Optical Instrumentation Engineers (SPIE) Conference Series, Vol. 7735, Ground-based and Airborne Instrumentation for Astronomy III, ed. I. S. McLean, S. K. Ramsay, \& H. Takami, 773508

Bailin, J., Kawata, D., Gibson, B. K., et al. 2005, ApJ, 627, L17

Barnes, J. \& Efstathiou, G. 1987, ApJ, 319, 575

Becklin, E. E. \& Neugebauer, G. 1968, ApJ, 151, 145

Behroozi, P., Hearin, A., \& Moster, B. P. 2021, arXiv e-prints, arXiv:2101.05280

Behroozi, P., Wechsler, R. H., Hearin, A. P., \& Conroy, C. 2019, MNRAS, 1134

Behroozi, P. S., Wechsler, R. H., \& Conroy, C. 2013, ApJ, 770, 57

Belokurov, V., Erkal, D., Evans, N. W., Koposov, S. E., \& Deason, A. J. 2018, MNRAS, 478, 611
Benítez-Llambay, A., Frenk, C. S., Ludlow, A. D., \& Navarro, J. F. 2019, MNRAS, 488, 2387

Bensby, T., Feltzing, S., \& Lundström, I. 2003, A\&A, 410, 527

Benson, A. J. 2017, MNRAS, 471, 2871

Berg, D. A., Skillman, E. D., Marble, A. R., et al. 2012, ApJ, 754, 98 Bernardi, M., Meert, A., Sheth, R. K., et al. 2013, MNRAS, 436, 697 Bett, P., Eke, V., Frenk, C. S., Jenkins, A., \& Okamoto, T. 2010, MNRAS, 404, 1137

Bland-Hawthorn, J. \& Gerhard, O. 2016, ARA\&A, 54, 529

Blank, M., Macciò, A. V., Dutton, A. A., \& Obreja, A. 2019, MNRAS, 487, 5476

Blumenthal, G. R., Faber, S. M., Flores, R., \& Primack, J. R. 1986, ApJ, 301, 27

Bond, J. R., Kofman, L., \& Pogosyan, D. 1996, Nature, 380, 603

Boylan-Kolchin, M., Besla, G., \& Hernquist, L. 2011, MNRAS, 414, 1560

Brook, C. B., Governato, F., Roškar, R., et al. 2011, MNRAS, 415, 1051

Brook, C. B., Stinson, G., Gibson, B. K., et al. 2012, MNRAS, 419, 771

Buck, T. 2020, MNRAS, 491, 5435

Buck, T., Macciò, A. V., Dutton, A. A., Obreja, A., \& Frings, J. 2019a, MNRAS, 483, 1314

Buck, T., Ness, M., Obreja, A., Macciò, A. V., \& Dutton, A. A. 2019b, ApJ, 874, 67

Buck, T., Obreja, A., Macciò, A. V., et al. 2020, MNRAS, 491, 3461

Buck, T., Rybizki, J., Buder, S., et al. 2021, arXiv e-prints, arXiv:2103.03884

Bullock, J. S., Dekel, A., Kolatt, T. S., et al. 2001, ApJ, 555, 240

Bundy, K., Bershady, M. A., Law, D. R., et al. 2015, ApJ, 798, 7

Callingham, T. M., Cautun, M., Deason, A. J., et al. 2019, MNRAS, 484, 5453

Catelan, P. \& Theuns, T. 1996, MNRAS, 282, 455

Cautun, M., Benítez-Llambay, A., Deason, A. J., et al. 2020, MNRAS, 494, 4291

Cen, R. \& Ostriker, J. 1992, ApJ, 393, 22

Chabrier, G. 2003, PASP, 115, 763

Chan, T. K., Kereš, D., Oñorbe, J., et al. 2015, MNRAS, 454, 2981

Codis, S., Pichon, C., Devriendt, J., et al. 2012, MNRAS, 427, 3320

Codis, S., Pichon, C., \& Pogosyan, D. 2015, MNRAS, 452, 3369

Cole, S. \& Lacey, C. 1996, MNRAS, 281, 716

Conroy, C. \& Wechsler, R. H. 2009, ApJ, 696, 620

Conroy, C., Wechsler, R. H., \& Kravtsov, A. V. 2007, ApJ, 668, 826

Croom, S. M., Lawrence, J. S., Bland-Hawthorn, J., et al. 2012, MNRAS, 421, 872

Dalcanton, J. J., Spergel, D. N., \& Summers, F. J. 1997, ApJ, 482, 659

De Silva, G. M., Freeman, K. C., Bland-Hawthorn, J., et al. 2015, MNRAS, 449, 2604

Debattista, V. P., van den Bosch, F. C., Roškar, R., et al. 2015, MNRAS, 452, 4094

Deng, L.-C., Newberg, H. J., Liu, C., et al. 2012, Research in Astronomy and Astrophysics, 12, 735

Desmond, H., Mao, Y.-Y., Wechsler, R. H., Crain, R. A., \& Schaye, J. 2017, MNRAS, 471, L11

Di Cintio, A., Brook, C. B., Dutton, A. A., et al. 2017, MNRAS, 466, L1

Di Cintio, A., Brook, C. B., Macciò, A. V., et al. 2014, MNRAS, 437, 415

Doménech-Moral, M., Martínez-Serrano, F. J., Domínguez-Tenreiro, R., \& Serna, A. 2012, MNRAS, 2503

D’Onghia, E. \& Fox, A. J. 2016, ARA\&A, 54, 363

Doroshkevich, A. G. 1970, Astrofizika, 6, 581

Du, M., Ho, L. C., Zhao, D., et al. 2019, ApJ, 884, 129

Dutton, A. A., Buck, T., Macciò, A. V., et al. 2020, MNRAS, 499, 2648

Dutton, A. A. \& Macciò, A. V. 2014, MNRAS, 441, 3359

Dutton, A. A., Macciò, A. V., Buck, T., et al. 2019a, MNRAS, 486, 655

Dutton, A. A., Macciò, A. V., Dekel, A., et al. 2016a, MNRAS, 461, 2658

Dutton, A. A., Macciò, A. V., Frings, J., et al. 2016b, MNRAS, 457, L74

Dutton, A. A., Obreja, A., \& Macciò, A. V. 2019b, MNRAS, 482, 5606

Dutton, A. A., Obreja, A., Wang, L., et al. 2017, MNRAS, 467, 4937

Dutton, A. A. \& van den Bosch, F. C. 2009, in Astronomical Society of the Pacific Conference Series, Vol. 419, Galaxy Evolution: Emerging Insights and Future Challenges, ed. S. Jogee, I. Marinova, L. Hao, \& G. A. Blanc, 355

Dutton, A. A., van den Bosch, F. C., Faber, S. M., et al. 2011, MNRAS, 410, 1660

Efstathiou, G., Frenk, C. S., White, S. D. M., \& Davis, M. 1988, MNRAS, 235, 715

Eilers, A.-C., Hogg, D. W., Rix, H.-W., et al. 2020, ApJ, 900, 186

Eilers, A.-C., Hogg, D. W., Rix, H.-W., \& Ness, M. K. 2019, ApJ, 871, 120

Elmegreen, B. G., Elmegreen, D. M., Tompkins, B., \& Jenks, L. G. 2017, ApJ, 847,14

Eric Jones, Travis Oliphant, P. P. et al. 2001, SciPy: Open source scientific tools for Python

Fall, S. M. \& Efstathiou, G. 1980, MNRAS, 193, 189

Fall, S. M. \& Romanowsky, A. J. 2018, ApJ, 868, 133

Firmani, C. \& Avila-Reese, V. 2009, MNRAS, 396, 1675

Fraternali, F. \& Binney, J. J. 2006, MNRAS, 366, 449

Fuhrmann, K. 1998, A\&A, 338, 161

Gaia Collaboration, Prusti, T., de Bruijne, J. H. J., et al. 2016, A\&A, 595, A1 
Gallazzi, A., Charlot, S., Brinchmann, J., White, S. D. M., \& Tremonti, C. A 2005, MNRAS, 362, 41

Ganeshaiah Veena, P., Cautun, M., van de Weygaert, R., Tempel, E., \& Frenk, C. S. 2021, MNRAS, 503, 2280

Garrison-Kimmel, S., Hopkins, P. F., Wetzel, A., et al. 2018, MNRAS, 481, 4133 Gill, S. P. D., Knebe, A., \& Gibson, B. K. 2004, MNRAS, 351, 399

Gilmore, G., Randich, S., Asplund, M., et al. 2012, The Messenger, 147, 25

Gilmore, G. \& Reid, N. 1983, MNRAS, 202, 1025

Gilmore, G., Wyse, R. F. G., \& Jones, J. B. 1995, AJ, 109, 1095

González, R. E., Kravtsov, A. V., \& Gnedin, N. Y. 2013, ApJ, 770, 96

Governato, F., Zolotov, A., Pontzen, A., et al. 2012, MNRAS, 422, 1231

Grand, R. J. J., Gómez, F. A., Marinacci, F., et al. 2017, MNRAS, 467, 179

Grand, R. J. J., van de Voort, F., Zjupa, J., et al. 2019, MNRAS, 490, 4786

Gravity Collaboration, Abuter, R., Amorim, A., et al. 2018, A\&A, 615, L15

Gunn, J. E. \& Gott, J. Richard, I. 1972, ApJ, 176, 1

Guo, Q., White, S., Li, C., \& Boylan-Kolchin, M. 2010, MNRAS, 404, 1111

Gurbatov, S. N., Saichev, A. I., \& Shandarin, S. F. 2012, Physics Uspekhi, 55, 223

Haardt, F. \& Madau, P. 2012, ApJ, 746, 125

Hammersley, P. L., Garzón, F., Mahoney, T. J., López-Corredoira, M., \& Torres, M. A. P. 2000, MNRAS, 317, L45

Haywood, M., Di Matteo, P., Lehnert, M. D., Katz, D., \& Gómez, A. 2013, A\&A, 560, A109

Helmi, A., Babusiaux, C., Koppelman, H. H., et al. 2018, Nature, 563, 85

Hogg, D. W., Eilers, A.-C., \& Rix, H.-W. 2019, AJ, 158, 147

Hoyle, F. 1951, in Problems of Cosmical Aerodynamics, 195

Hunter, J. D. 2007, Computing in Science and Engineering, 9, 90

Jiang, F., Dekel, A., Kneller, O., et al. 2019, MNRAS, 488, 4801

Jurić, M., Ivezić, Ž., Brooks, A., et al. 2008, ApJ, 673, 864

Katz, N. 1992, ApJ, 391, 502

Katz, N. \& Gunn, J. E. 1991, ApJ, 377, 365

Katz, N., Hernquist, L., \& Weinberg, D. H. 1992, ApJ, 399, L109

Kelz, A., Verheijen, M. A. W., Roth, M. M., et al. 2006, PASP, 118, 129

Kewley, L. J. \& Ellison, S. L. 2008, ApJ, 681, 1183

Kirby, E. N., Cohen, J. G., Guhathakurta, P., et al. 2013, ApJ, 779, 102

Knollmann, S. R. \& Knebe, A. 2009, ApJS, 182, 608

Kravtsov, A. V., Vikhlinin, A. A., \& Meshcheryakov, A. V. 2018, Astronomy Letters, 44, 8

Kuijken, K. \& Gilmore, G. 1991, ApJ, 367, L9

Lang, P., Wuyts, S., Somerville, R. S., et al. 2014, ApJ, 788, 11

Lelli, F., McGaugh, S. S., \& Schombert, J. M. 2016, AJ, 152, 157

Libeskind, N. I., Carlesi, E., Grand, R. J. J., et al. 2020, MNRAS, 498, 2968

López, P., Cautun, M., Paz, D., Merchán, M., \& van de Weygaert, R. 2021, MNRAS, 502, 5528

Macciò, A. V., Dutton, A. A., \& van den Bosch, F. C. 2008, MNRAS, 391, 1940

Macciò, A. V., Udrescu, S. M., Dutton, A. A., et al. 2016, MNRAS, 463, L69

Majewski, S. R., Schiavon, R. P., Frinchaboy, P. M., et al. 2017, AJ, 154, 94

McConnachie, A. W. 2012, AJ, 144, 4

McMillan, P. J. 2017, MNRAS, 465, 76

Mo, H. J., Mao, S., \& White, S. D. M. 1998, MNRAS, 295, 319

Moster, B. P., Naab, T., \& White, S. D. M. 2013, MNRAS, 428, 3121

Moster, B. P., Naab, T., \& White, S. D. M. 2018, MNRAS, 477, 1822

Moster, B. P., Somerville, R. S., Maulbetsch, C., et al. 2010, ApJ, 710, 903

Navarro, J. F., Frenk, C. S., \& White, S. D. M. 1997, ApJ, 490, 493

Navarro, J. F. \& White, S. D. M. 1994, MNRAS, 267, 401

Obreja, A., Dutton, A. A., Macciò, A. V., et al. 2019, MNRAS, 487, 4424

Obreja, A., Macciò, A. V., Moster, B., et al. 2018, MNRAS, 477, 4915

Obreja, A., Stinson, G. S., Dutton, A. A., et al. 2016, MNRAS, 459, 467

Obreschkow, D. \& Glazebrook, K. 2014, ApJ, 784, 26

Okuda, H., Maihara, T., Oda, N., \& Sugiyama, T. 1977, Nature, 265, 515

Panter, B., Jimenez, R., Heavens, A. F., \& Charlot, S. 2008, MNRAS, 391, 1117

Peacock, J. A. \& Smith, R. E. 2000, MNRAS, 318, 1144

Pedregosa, F., Varoquaux, G., Gramfort, A., \& et al. 2011, Journal of Machine Learning Research, 12, 2825

Peebles, P. J. E. 1965, ApJ, 142, 1317

Peebles, P. J. E. 1971, A\&A, 11, 377

Peebles, P. J. E. 1982, ApJ, 263, L1

Peschken, N., Athanassoula, E., \& Rodionov, S. A. 2017, MNRAS, 468, 994

Peterson, P. 2009, International Journal of Computational Science and Engineering, 4, 296

Planck Collaboration. 2014, A\&A, 571, A16

Planck Collaboration, Aghanim, N., Akrami, Y., et al. 2020, A\&A, 641, A1

Pontzen, A., Roškar, R., Stinson, G., \& Woods, R. 2013, pynbody: N-Body/SPH analysis for python, Astrophysics Source Code Library

Portail, M., Gerhard, O., Wegg, C., \& Ness, M. 2017, MNRAS, 465, 1621

Posti, L., Fraternali, F., Di Teodoro, E. M., \& Pezzulli, G. 2018, A\&A, 612, L6

Reddy, B. E., Lambert, D. L., \& Allende Prieto, C. 2006, MNRAS, 367, 1329

Reid, M. J. \& Brunthaler, A. 2004, ApJ, 616, 872

Rodriguez-Gomez, V., Sales, L. V., Genel, S., et al. 2017, MNRAS, 467, 3083

Romanowsky, A. J. \& Fall, S. M. 2012, ApJS, 203, 17
Sachs, R. K. \& Wolfe, A. M. 1967, ApJ, 147, 73

Sandage, A. 1961, The Hubble Atlas of Galaxies

Santos-Santos, I. M. E., Fattahi, A., Sales, L. V., \& Navarro, J. F. 2021, MNRAS, 504,4551

Sawala, T., Frenk, C. S., Fattahi, A., \& et al. 2016, MNRAS, 457, 1931

Schaye, J., Crain, R. A., Bower, R. G., et al. 2015, MNRAS, 446, 521

Schwarz, G. 1978, Annals of Statistics, 6, 461

Schwarzschild, M. 1979, ApJ, 232, 236

Searle, L. \& Zinn, R. 1978, ApJ, 225, 357

Sellwood, J. A. \& Binney, J. J. 2002, MNRAS, 336, 785

Shao, S., Cautun, M., Frenk, C. S., et al. 2016, MNRAS, 460, 3772

Shen, S., Wadsley, J., \& Stinson, G. 2010, MNRAS, 407, 1581

Silk, J. 1968, ApJ, 151, 459

Skrutskie, M. F., Cutri, R. M., Stiening, R., et al. 2006, AJ, 131, 1163

Somerville, R. S., Barden, M., Rix, H.-W., et al. 2008, ApJ, 672, 776

Soubiran, C., Bienaymé, O., \& Siebert, A. 2003, A\&A, 398, 141

Steinmetz, M., Zwitter, T., Siebert, A., et al. 2006, AJ, 132, 1645

Stinson, G., Seth, A., Katz, N., et al. 2006, MNRAS, 373, 1074

Stinson, G. S., Brook, C., Macciò, A. V., et al. 2013, MNRAS, 428, 129

Syer, D. \& Tremaine, S. 1996, MNRAS, 282, 223

Teklu, A. F., Remus, R.-S., Dolag, K., et al. 2015, ApJ, 812, 29

Thielemann, F.-K., Nomoto, K., \& Yokoi, K. 1986, A\&A, 158, 17

Tollet, E., Macciò, A. V., Dutton, A. A., et al. 2016, MNRAS, 456, 3542

Vale, A. \& Ostriker, J. P. 2004, MNRAS, 353, 189

van den Bosch, F. C., Yang, X., \& Mo, H. J. 2003, MNRAS, 340, 771

Wadsley, J. W., Keller, B. W., \& Quinn, T. R. 2017, MNRAS, 471, 2357

Wadsley, J. W., Veeravalli, G., \& Couchman, H. M. P. 2008, MNRAS, 387, 427

Walt, S. v. d., Colbert, S. C., \& Varoquaux, G. 2011, Computing in Science and Engineering, 13, 22

Wang, L., Dutton, A. A., Stinson, G. S., et al. 2015, MNRAS, 454, 83

Wang, W., Takada, M., Li, X., et al. 2021, MNRAS, 500, 3776

Wegg, C., Gerhard, O., \& Portail, M. 2015, MNRAS, 450, 4050

Wes McKinney. 2010, in Proceedings of the 9th Python in Science Conference, ed. Stéfan van der Walt \& Jarrod Millman, 56 - 61

White, S. D. M. 1984, ApJ, 286, 38

White, S. D. M. \& Frenk, C. S. 1991, ApJ, 379, 52

White, S. D. M. \& Rees, M. J. 1978, MNRAS, 183, 341

Woosley, S. E. \& Weaver, T. A. 1995, ApJS, 101, 181

Wright, A. H., Robotham, A. S. G., Driver, S. P., et al. 2017, MNRAS, 470, 283

Wright, E. L., Eisenhardt, P. R. M., Mainzer, A. K., et al. 2010, AJ, 140, 1868

Yanny, B., Rockosi, C., Newberg, H. J., et al. 2009, AJ, 137, 4377

Zahid, H. J., Geller, M. J., Kewley, L. J., et al. 2013, ApJ, 771, L19

Zel'Dovich, Y. B. 1970, A\&A, 500, 13

Zoccali, M., Gonzalez, O. A., Vasquez, S., et al. 2014, A\&A, 562, A66 


\section{Appendix A: MW-like galaxies in NIHAO}

Figure A.1 shows the circular velocity profiles (thick gray lines) in the equatorial plane $V_{\mathrm{c}}=\sqrt{R \partial \Phi / \partial R}$ for the five NIHAO galaxies that have stellar and DM masses within the ranges estimated for the MW. The galaxy with the most massive halo among the five, g8.26e11, shown in the top panel, has also the closest $\lambda=0.057$ to $\lambda_{\mathrm{MW}}=0.061$ for the contracted NFW model. This simulated galaxy has been shown to resamble MW in many aspects: i) thin- and thick-disk properties in terms of scale lengths and rotational velocity profiles (Obreja et al.2018); ii) vertical velocity dispersion vs stellar age and iii) thin- and thick-disk scale heights at $R_{\odot}$ (Buck et al.2020); iv) bimodal 2D distribution in $[\alpha / \mathrm{Fe}]$ vs $[\mathrm{Fe} / \mathrm{H}]$ at $R_{\odot}(\mathrm{Buck} 2020)$, and v) satellites distribution and properties (Buck et al. 2019a). From the stellar and DM contributions to the $V_{\mathrm{c}}(R)$ of this galaxy (dashed red and solid black curves), it can be appreciated that DM becomes the dominant term at $R \simeq 8 \mathrm{kpc}$, similar to what Portail et al. (2017) found from their dynamical model of MW, which includes the bar.

The two main differences between g8.26e11 and our Galaxy is that the simulation has no bar or only a very weak bar, and it does not have satellites as massive as LMC and SMC (Buck et al.2019a).

\section{Appendix B: MW's rotational velocities}

Table B.1 gives $v_{\phi}(R)$ for the two disks of the Galaxy. These data have been used in Fig. 4 and in the fit of Eq. 5 .

Table B.1. Rotational velocities profiles of MW's thin and thick disks.

\begin{tabular}{ccc}
\hline$R[\mathrm{kpc}]$ & $v_{\phi \text {,thin }}\left[\mathrm{km} \mathrm{s}^{-1}\right]$ & $v_{\phi \text {,thick }}\left[\mathrm{km} \mathrm{s}^{-1}\right]$ \\
\hline 5.25 & $218.85 \pm 1.77$ & $193.35 \pm 2.92$ \\
5.75 & $223.81 \pm 1.24$ & $195.50 \pm 3.10$ \\
6.25 & $223.60 \pm 1.41$ & $194.11 \pm 3.16$ \\
6.75 & $221.72 \pm 1.31$ & $195.87 \pm 3.00$ \\
7.25 & $221.01 \pm 1.06$ & $190.51 \pm 2.78$ \\
7.75 & $221.60 \pm 0.69$ & $188.01 \pm 1.84$ \\
8.25 & $221.62 \pm 0.77$ & $185.15 \pm 2.21$ \\
8.75 & $218.64 \pm 0.84$ & $191.17 \pm 3.32$ \\
9.25 & $219.30 \pm 0.55$ & $182.92 \pm 2.84$ \\
9.75 & $217.18 \pm 0.47$ & $184.83 \pm 2.52$ \\
10.25 & $216.93 \pm 0.50$ & $184.33 \pm 2.90$ \\
10.75 & $216.34 \pm 0.45$ & $181.89 \pm 2.78$ \\
11.25 & $215.44 \pm 0.47$ & $179.06 \pm 3.51$ \\
11.75 & $215.63 \pm 0.49$ & $176.38 \pm 3.16$ \\
12.25 & $214.46 \pm 0.48$ & $181.83 \pm 4.55$ \\
12.75 & $213.06 \pm 0.50$ & $185.16 \pm 4.14$ \\
13.25 & $211.58 \pm 0.57$ & - \\
13.75 & $209.79 \pm 0.57$ & - \\
14.25 & $207.37 \pm 0.65$ & - \\
14.75 & $206.79 \pm 0.85$ & - \\
15.25 & $204.59 \pm 1.09$ & - \\
15.75 & $205.78 \pm 1.41$ & - \\
16.25 & $203.89 \pm 1.73$ & - \\
16.75 & $201.61 \pm 1.73$ & - \\
17.25 & $203.03 \pm 1.66$ & - \\
17.75 & $202.81 \pm 1.86$ & - \\
18.25 & $202.26 \pm 2.37$ & - \\
18.75 & $193.71 \pm 2.35$ & - \\
19.25 & $196.02 \pm 3.02$ & - \\
19.75 & $191.09 \pm 4.40$ & - \\
\hline & &
\end{tabular}

Article number, page 14 of 15

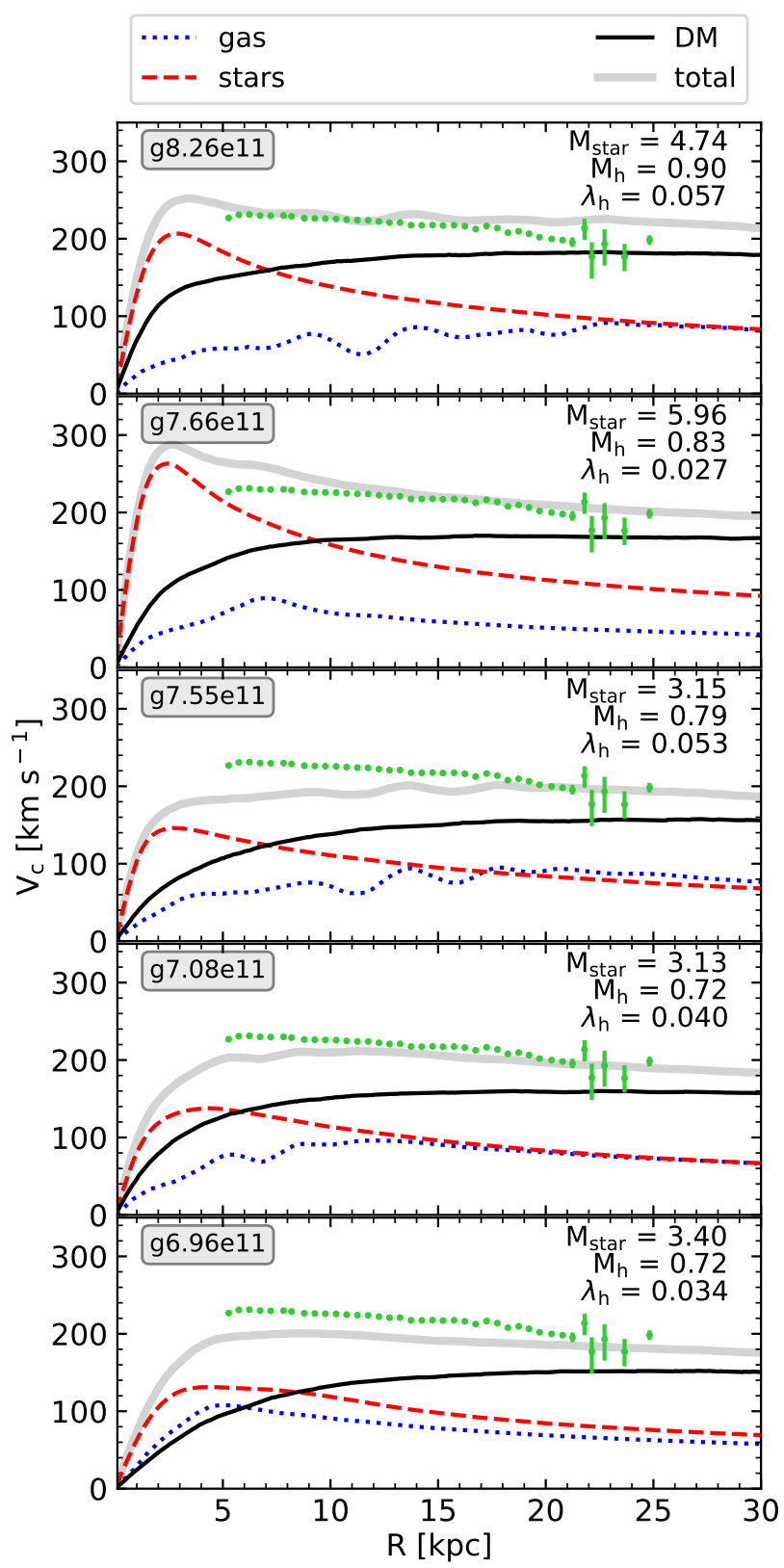

Fig. A.1. Circular velocity profiles of MW-like galaxies in NIHAO. DM halo mass, $M_{\mathrm{h}}$, in units of $10^{12} \mathrm{M}_{\odot}$, halo spin, and total stellar mass, $M_{\text {star }}$, in units of $10^{10} \mathrm{M}_{\odot}$ are quoted in the top right of each panel. MW observational data (green points) are from Eilers et al. (2019).

\section{Appendix C: MW mass model}

The free parameters of the MW mass model of Cautun et al. (2020), which we needed for computing the AM of the thin and thick disks, are given in Table C.1. The uncertainties on the parameters have been symmetrized such that we can use a use a six-variate Gaussian distribution with a given covariance matrix to approximate the model of Cautun et al. The most important correlations in these models are among the DM halo mass $M_{\mathrm{h}}$, and thin and thick disk masses, $M_{\text {thin }}$ and $M_{\text {thick }}$, as can appreciated from their Fig. 12. 
For the contracted NFW model, we found the following covariance matrix:

$\operatorname{cov}=\left(\begin{array}{cccccc}\sigma_{1}^{2} & 0.7 \sigma_{1} \sigma_{2} & 0.6 \sigma_{1} \sigma_{3} & 0 & 0 & 0 \\ 0.7 \sigma_{1} \sigma_{2} & \sigma_{2}^{2} & 0.8 \sigma_{2} \sigma_{3} & 0 & 0 & 0 \\ 0.6 \sigma_{1} \sigma_{3} & 0.8 \sigma_{2} \sigma_{3} & \sigma_{3}^{2} & 0 & 0 & 0 \\ 0 & 0 & 0 & \sigma_{4}^{2} & 0 & 0 \\ 0 & 0 & 0 & 0 & \sigma_{5}^{2} & 0 \\ 0 & 0 & 0 & 0 & 0 & \sigma_{6}^{2}\end{array}\right)$

to reproduce well the 68 percentile contours in the panels $M_{\text {thin }}$ versus $\log \left(M_{\mathrm{h}}\right), M_{\text {thick }}$ versus $\log \left(M_{\mathrm{h}}\right)$, and $M_{\text {thin }}$ vs $M_{\text {thick }}$ of Fig. 12 in Cautun et al. We neglect the weak correlations with the other parameters. The corresponding indices of each parameter are listed in the last column of Table C.1.

For the uncontracted NFW model, we use instead the following covariance matrix:

$\operatorname{cov}=\left(\begin{array}{cccccc}\sigma_{1}^{2} & 0.7 \sigma_{1} \sigma_{2} & 0.6 \sigma_{1} \sigma_{3} & 0 & 0 & 0 \\ 0.7 \sigma_{1} \sigma_{2} & \sigma_{2}^{2} & 0.7 \sigma_{2} \sigma_{3} & 0 & 0 & 0 \\ 0.6 \sigma_{1} \sigma_{3} & 0.7 \sigma_{2} \sigma_{3} & \sigma_{3}^{2} & 0 & 0 & 0 \\ 0 & 0 & 0 & \sigma_{4}^{2} & 0 & 0 \\ 0 & 0 & 0 & 0 & \sigma_{5}^{2} & 0 \\ 0 & 0 & 0 & 0 & 0 & \sigma_{6}^{2}\end{array}\right)$

Table C.1. Parameters of the MW mass models of Cautun et al. (2020). Here, $M_{\text {gas }}$ sums up the disk (HI + molecular) and the CGM, both contributions being kept fixed in the mass models. The bulge and gas masses are needed to compute the total viral mass $M_{200}$ in Eq. 6

\begin{tabular}{cccc}
\hline \hline Parameter & contracted NFW & NFW & index \\
\hline $\log \left(M_{\mathrm{h}} / \mathrm{M}_{\odot}\right)$ & $11.987 \pm 0.095$ & $11.914 \pm 0.076$ & 1 \\
$M_{\text {thin }}\left[10^{10} \mathrm{M}_{\odot}\right]$ & $3.18 \pm 0.38$ & $3.98 \pm 0.47$ & 2 \\
$M_{\text {thick }}\left[10^{10} \mathrm{M}_{\odot}\right]$ & $0.92 \pm 0.16$ & $1.07 \pm 0.19$ & 3 \\
$R_{\text {thin }}[\mathrm{kpc}]$ & $2.63 \pm 0.13$ & $2.43 \pm 0.11$ & 4 \\
$R_{\text {thick }}[\mathrm{kpc}]$ & $3.80 \pm 0.72$ & $3.88 \pm 0.65$ & 5 \\
$M_{\text {bulge }}\left[10^{10} \mathrm{M}_{\odot}\right]$ & $0.94 \pm 0.09$ & $0.92 \pm 0.09$ & 6 \\
$M_{\text {gas }}\left[10^{10} \mathrm{M}_{\odot}\right]$ & 7.6 & 6.7 & - \\
\hline
\end{tabular}

